\title{
Multipoint High-Fidelity Aerostructural Optimization of a Transport Aircraft Configuration
}

\author{
Gaetan K. W. Kenway* and Joaquim R. R. A. Martins \\ University of Michigan, Ann Arbor, Michigan 48109
}

DOI: $10.2514 / 1 . C 032150$

\begin{abstract}
This paper presents multipoint high-fidelity aerostructural optimizations of a long-range wide-body transonic transport aircraft configuration. The aerostructural analysis employs Euler computational fluid dynamics with a 2-million-cell mesh and a structural finite-element model with 300,000 degrees of freedom. The coupled adjoint sensitivity method is used to efficiently compute gradients, enabling the use of gradient-based optimization with respect to hundreds of aerodynamic shape and structural sizing variables. The NASA Common Research Model is used as the baseline configuration, together with a wing box structure that was designed for this study. Two design optimization problems are solved: one where takeoff gross weight is minimized, and another where fuel burn is minimized. Each optimization uses a multipoint formulation with five cruise conditions and two maneuver conditions. Each of the optimization problems have 476 design variables, including wing planform, airfoil shape, and structural thickness variables. Optimized results are obtained within $36 \mathrm{~h}$ of wall time using 435 processors. The resulting optimal configurations are discussed and analyzed for the aerostructural tradeoffs resulting from each objective. The takeoff gross weight minimization results in a $4.2 \%$ reduction in takeoff gross weight with a $6.6 \%$ fuel burn reduction, whereas the fuel-burn optimization resulted in an $11.2 \%$ fuel burn reduction with no significant change in the takeoff gross weight.
\end{abstract}

\section{Nomenclature}

$\mathcal{A}=$ aerodynamic residuals

$A_{\text {ref }} \quad=$ reference wing area

$A_{\text {wet }}=$ wetted surface area

$C_{f} \quad=$

$C_{M} \quad=$

$c_{T} \quad=$

$D, C_{D}$

I

$L, C_{L}$

M

$R$

$\mathcal{S}$

$t / c$

$u$

V

$W_{1}$

$W_{2}$

$W_{f}$

$w$

$x$

$\alpha$

$\beta$

$\gamma$

$\phi$

$\psi$

\section{turbulent skin friction coefficient}

coefficient of moment

thrust specific fuel consumption

drag, coefficient of drag

function of interest

form factor correction

lift, coefficient of lift

Mach number

aircraft range

structural residuals

thickness-to-chord ratio

structural state vector

aircraft flight speed

initial cruise weight

final cruise weight

fuel burn

aerodynamic state vector

design variable vector

angle of attack

objective sensitivity with respect to $W_{2}$

objective sensitivity with respect to $C_{D}$

structural adjoint vector

aerodynamic adjoint vector

\section{Introduction}

$\mathbf{T}$ HE design of aircraft has benefited greatly from the use of numerical optimization techniques $[\underline{1}, 2]$, and the need to include the relevant disciplines in such design problems led to the field of multidisciplinary design optimization (MDO) [2,3]. In particular, the aerodynamic design of wings and their internal structure have been the subject of MDO efforts for a considerable time because aerodynamics and structures are two of the most tightly coupled disciplines in wing design.

Coupling aerodynamic and structural numerical models to compute the static aeroelastic shape of aircraft wings is essential when designing wings that are flexible. Even small changes in aerodynamic shape can have a large effect on the aerodynamic performance, and multiple flow conditions result in multiple shapes. This is particularly important for swept wings, where bending-twist coupling can result in large changes in the twist distribution [4]. The analysis of static aeroelastic shapes was introduced as soon as the first simple numerical models for aerodynamics and structures matured. Brown [5], for example, coupled a lifting-line model to a beamtheory model to obtain the flying shape of a swept wing, and earlier work did the same with even simpler models [6]. In recent years, this coupling has become even more important because the trend has been to increase the aspect ratio of aircraft wings, making them more flexible. Wing flexibility impacts not only the static flying shape of the wing but also its dynamics, resulting in aeroelastic phenomena such as flutter and aileron reversal [7].

In the present work, we restrict ourselves to the optimization of the static aeroelastic shape. We refer to the simultaneous optimization of aerodynamic shape and structural sizing as aerostructural optimization. By construction, aerostructural optimization performs optimal static aeroelastic tailoring. One of the main characteristics of this tailoring is that it takes advantage of the wing deflection at various flight conditions by shifting the spanwise lift distribution inboard at the critical load conditions, while maintaining a lift distribution with lower induced drag at cruise conditions.

One of the earliest efforts in aerostructural optimization was by Haftka [ $\underline{8}$, who combined a lifting-line aerodynamic model with a simple structural finite-element analysis to iteratively obtain the flying shape due to the structural deformations. The tradeoffs between weight and induced drag were quantified for both aluminium and composite configurations. Grossman et al. [9] investigated the complete aerostructural analysis and optimization of a sailplane. They showed that integrated aerostructural optimization 
gave higher performance designs than those found by sequential optimization (aerodynamic optimization followed by structural minimization). They subsequently considered the design of a subsonic, forward-swept transport aircraft wing [10]. This failure of sequential optimization to produce the optimal result is further explained by Chittick and Martins [11]. When performing sequential optimization, the optimizer does not have the information necessary for optimal aeroelastic tailoring. This is because the aerodynamic optimization does not account for the structural benefit of shifting the lift distribution inboard, and the structural optimization does not tailor the sizing to produce a deflected wing that is aerodynamically favorable.

With the advent of higher-fidelity modeling in both structures and aerodynamics, numerical optimization has been extensively applied to each of these disciplines separately. On the structures side, since Schmit [12] first proposed the application of numerical optimization to structural design, increasingly detailed finite-element models have been used in wing structural sizing optimization $[13,14]$. Several commercial software packages are able to perform such optimizations. The increase in fidelity in the structural model is required for a more refined sizing of the structure under complex structural failure constraints, leading to a better estimate of the optimized structural weight.

The fidelity of the models used for aerodynamic shape optimization has also been increasing, and it is now possible to use computational fluid dynamics (CFD) to optimize a design with respect to hundreds of design variables using both Euler [15-18] and Navier-Stokes models [19-22]. In the design of transonic wings, it is particularly important to use high-fidelity models to correctly predict the drag due to viscous and compressibility effects. However, aerodynamic shape optimization alone is insufficient for wing design because it is impossible to perform the tradeoffs for wing thickness, span, and sweep, which require a model of how the wing weight varies with respect to these parameters. Furthermore, the shape resulting from an aerodynamic optimization must then be matched by designing a structure that deflects to the desired shape under the given aerodynamic loads, which results in a suboptimal wing from the multidisciplinary point of view.

To take advantage of the higher-fidfelity models, it is desirable to optimize with respect to large numbers of variables. On the aerodynamic side, for example, airfoil shape variables distributed both chordwise and spanwise are required to reduce the drag of a wing in an effective way. When it comes to the structures, having more sizing variables decreases the minimum weight that can be achieved. To handle the large numbers of design variables, the efforts described previously employed gradient-based optimization algorithms together with adjoint methods to compute the required gradients efficiently. Adjoint methods are able to compute gradients with respect to large numbers of design variables with a cost that is comparable to the cost of solving the corresponding model. Since the 1960 s, these methods have been known in the structural optimization community [23] and have been adopted by aerodynamic shape optimization researchers [15,24,25].

Given the importance of coupling the aerodynamics and the structures in wing design, the coupling of high-fidelity versions of these models for design optimization is a natural extension of the efforts described previously. Various techniques have been proposed over the years for coupling CFD to computational structural mechanics (CSM) solvers, with contributions in load and displacement transfer schemes [26-29] and solution techniques for solving the coupled system of equations [30,31].

To enable high-fidelity aerostructural optimization with respect to large numbers of design variables, Martins et al. $[\underline{32}, 33]$ proposed the use of a coupled adjoint method for aerostructural design optimization using Euler CFD and linear finite-element analysis in a two-field formulation. Then, they applied this method to the aerostructural design of a supersonic business jet with respect to 97 shape and sizing variables [34]. Although that work demonstrated that the coupled adjoint method was feasible, several developments were needed to make this approach scalable and practical. These developments were made by Kenway et al. [35], who implemented a coupled Newton-Krylov approach to solve the aerostructural system, a Krylov approach to solve the coupled adjoint, new techniques to compute the partial derivatives in the coupled-adjoint systems, and other improvements that made this approach truly scalable. The coupled gradients of the aerodynamic and structural objective functions and the constraints were shown to be accurate and scalable to thousands of design variables and millions of degrees of freedom [35]. The authors also provided a detailed review of previous work on the aerostructural adjoint approach. For a broader review of methods for computing coupled derivatives, we refer the reader to Martins and Hwang [36].

A few other authors have implemented coupled adjoint methods for the aerostructural equations. Maute et al. [37] presented a coupled adjoint formulation using discrete-analytical derivatives, which was later improved [38]. Brezillon et al. [39] describe ongoing work at DRL (German Aerospace Center) toward high-fidelity aerostructural analysis and optimization capabilities. Ghazlane et al. [40] present a similar effort. In spite of the potential for the adjoint method to handle large numbers of variables, the number of design variables was very limited in all these efforts.

Some coupled-adjoint-based aerostructural optimization work has increased the fidelity of the structural analysis relative to previous work while lowering the fidelity of the aerodynamics to a panel code. Kennedy and Martins [41] used aerostructural optimization to compare metallic and composite wings while considering buckling constraints and including panel-level structural variables. They used a new technique for handling the composite laminate parametrization that successfully solved optimization problems with thousands of design variables [42], and they were able to examine the tradeoff trends between weight and drag for the two different materials.

Some researchers have carried out aerostructural optimization without the aid of the adjoint method. Piperni et al. [43] performed the preliminary optimization of a business jet in an industrial setting. They used a transonic small-disturbance code to predict the aerodynamic characteristics in the transonic flight regime and a finite-element model to compute the displacements, weight, and stresses. DeBlois and Abdo [44] used a similar framework to investigate the tradeoffs between metallic and composite materials. They considered many detailed aspects of the aircraft design process, but they used sequential optimization for some of the disciplines.

The objective of this work is to perform the aerostructural design optimization of a transport aircraft wing with an unprecedented level of fidelity, number of design variables, and number of flight conditions. We achieve this objective by leveraging our previous efforts toward making the coupled aerostructural analysis and derivative computations scalable in a high-performance parallel computing environment [35]. Having addressed the main challenges that prevented high-fidelity aerostructural optimization from realizing its full potential, we pursue the optimization of a conventional transonic aircraft configuration. We present multipoint optimizations that minimize two different objectives: takeoff gross weight and fuel burn. By optimizing with respect to all of the design variables simultaneously, we ensure that we find a true multidisciplinary optimum that minimizes the corresponding objective function, as opposed to a sequential suboptimal result $[10,11]$.

Although we use a conventional configuration, the proposed approach is expected to be even more useful when applied to the optimization of unconventional configurations, which are less well understood. We select a conventional transport configuration because this configuration has been refined over several decades, the MDO tradeoffs are well understood, and its optimization is not expected to diverge too much from a good baseline design. If the optimized configuration is similar to actual aircraft, then the high-fidelity analysis and the optimization problem formulation are likely to be capturing the multidisciplinary tradeoffs accurately. The optimization presented in this paper is a critical first step before we consider unconventional configurations with the same computational methods.

The remainder of this paper is organized as follows. Section II describes the aerostructural analysis and the framework used for the optimizations. This is just a brief description because this has been 
detailed in previous work [35]. Section III describes the optimization problem formulation, and Sec. IV presents the optimization results. Section $\mathrm{V}$ provides concluding remarks

\section{Computational Techniques}

In this section, we present a summary of the aerostructural analysis and derivative computation techniques used in this paper. A much more detailed description is presented in previous work by the authors, together with verification and benchmarking results [35].

\section{A. Aerostructural Analysis}

To capture the shock waves and the associated wave drag in transonic flow, we model the aerodynamics with the Euler equations. A Reynolds-averaged Navier-Stokes (RANS) model would be preferred, but the multipoint aerostructural optimization presented herein costs significantly more with RANS than with Euler [21,22]. We use a structured multiblock flow solver SUmb [45], and we solve the nonlinear aerodynamic equations using a preconditioned matrixfree Newton-Krylov approach.

The structural solver used in this work is the toolkit for the analysis of composite structures (TACS) by Kennedy and Martins [46]. For the thin-shell problems we encounter in wing structural models, it is possible to have matrix condition numbers that exceed $\mathcal{O}\left(10^{9}\right)$. For this reason, we use a parallel direct method to solve the structural governing equations.

The load and displacement transfer scheme that we use closely follows the work of Brown [26]. In this approach, rigid links consisting of the shortest distance between the aerodynamic nodes and the structural surface are used to extrapolate the displacements from the structural surface to the CFD surface. The consistent force vector is determined by employing the method of virtual work, ensuring that the force transfer is conservative. The integration of the forces occurs on the aerodynamic mesh and is transmitted back through the rigid links to the structure. The two primary advantages of this scheme are that it is consistent and conservative by construction, and that it may be used to transfer loads and displacements between aerodynamic and structural meshes that are not coincident.

The solution of the aerostructural system can be obtained by a fully coupled Newton-Krylov approach or a segregated Gauss-Seidel approach. The latter approach was used to produce the results presented in Sec. IV. More details on these approaches can be found in previous work $[\underline{33}, \underline{35}, \underline{46}]$.

\section{B. Viscous Drag Estimate}

Because we are using the Euler equations to model the flow over the aircraft, we are not able to evaluate the viscous drag contribution. However, for aerostructural optimization, an estimate of the total aircraft drag is required, including the viscous contribution. With viscous drag, the drag penalty associated with increasing wing area and decreasing wing chords would not be captured, resulting in unrealistic planforms. Therefore, we use a flat-plate turbulent skin friction estimate with form factor corrections to account for the added pressure drag due to viscous effects. The van Driest II method [47] is used to estimate the turbulent skin friction coefficient, $C_{f}$. For winglike bodies, we use the following form-factor correction [48], which is based on Torenbeek [49]:

$$
K_{\text {form }}=1+2.7\left(\frac{t}{c}\right)+100\left(\frac{t}{c}\right)^{4}
$$

where $t / c$ is the thickness-to-chord ratio of the wing section being considered. The form factor accounts for the higher flow velocities relative to the flat plate and corrects for the additional pressure drag due to the boundary-layer displacement effect. For fuselage-like bodies, the form-factor correction is

$$
K_{\text {form }}=1+1.5\left(\frac{d}{l}\right)^{1.5}+50\left(\frac{d}{l}\right)^{3}
$$

where $d / l$ is the ratio of diameter to length. The contribution of a given component to the drag coefficient is then

$$
C_{D}=K_{\text {form }} C_{f} \frac{A_{\text {wet }}}{A_{\text {ref }}}
$$

where $A_{\text {wet }}$ is the wetted surface area, and $A_{\text {ref }}$ is the reference wing area.

In our implementation, we use the three-dimensional surface geometries shown in Fig. 1, which allows us to use the same geometric design variables as in the aerodynamic and structural disciplines. The viscous drag for the wing and tail are computed in a stripwise fashion that accounts for local changes in the Reynolds number due to chord modifications and thickness-to-chord changes due to shape changes. Smooth Kreisselmeier-Steinhauser (KS) functions [50] are used to estimate the $t / c$ ratio from the discrete surface data to ensure smooth, continuous derivatives.

\section{Computation of Aerostructural Derivatives}

As discussed in the introduction, the efficient sensitivity analysis of high-fidelity aerostructural systems is a significant challenge. The coupled adjoint method developed $[33,35]$ is the key enabling method for the aerostructural optimizations presented in this work. The authors have previously demonstrated efficient scaling of the coupled adjoint method to aerostructural systems with over 80 million aerodynamic degrees of freedom and over 1 million structural degrees of freedom. The method has also been shown to scale to thousands of design variables [35]. A more general derivation of the theory behind the coupled adjoint method and its connection to other sensitivity analysis methods can be found in Martins and Hwang [36], and a much more detailed description of the aerostructural analysis and coupled adjoint implementation can be found in Kenway et al. [35].

For completeness, however, we present a brief description of the coupled adjoint method. We denote the residuals, state variables, and adjoint variables for the aerodynamic discipline as $\mathcal{A}, w, \psi$ and for the structural discipline as $\mathcal{S}, u, \phi$, respectively. First, we write the total sensitivity of a given function of interest $I$ :

$$
\frac{\mathrm{d} I}{\mathrm{~d} x}=\frac{\partial I}{\partial x}-\left[\begin{array}{ll}
\frac{\partial I}{\partial w} & \frac{\partial I}{\partial u}
\end{array}\right]\left[\begin{array}{l}
\frac{\mathrm{d} w}{\mathrm{~d} x} \\
\frac{\mathrm{d} u}{\mathrm{~d} x}
\end{array}\right]
$$

We make a distinction between the partial derivatives, denoted " $\partial$ ", and the total derivatives, denoted " $\mathrm{d}$ ". The computation of the total derivatives requires the solution of the nonlinear system of governing equations (i.e., the state variables are varied such that the residuals of the equations remain zero). The partial derivatives reflect the influence of the variables while keeping the state variables constant [35]. We write the total sensitivity of the residuals as

$$
\left[\begin{array}{l}
\frac{\mathrm{d} \mathcal{A}}{\mathrm{d} x} \\
\frac{\mathrm{d} \mathcal{S}}{\mathrm{d} x}
\end{array}\right]=\left[\begin{array}{l}
\frac{\partial \mathcal{A}}{\partial x} \\
\frac{\partial \mathcal{S}}{\partial x}
\end{array}\right]-\left[\begin{array}{ll}
\frac{\partial \mathcal{A}}{\partial w} & \frac{\partial \mathcal{A}}{\partial u} \\
\frac{\partial \mathcal{S}}{\partial w} & \frac{\partial \mathcal{S}}{\partial u}
\end{array}\right]\left[\begin{array}{l}
\frac{\mathrm{d} w}{\mathrm{~d} x} \\
\frac{\mathrm{d} u}{\mathrm{~d} x}
\end{array}\right]=0
$$

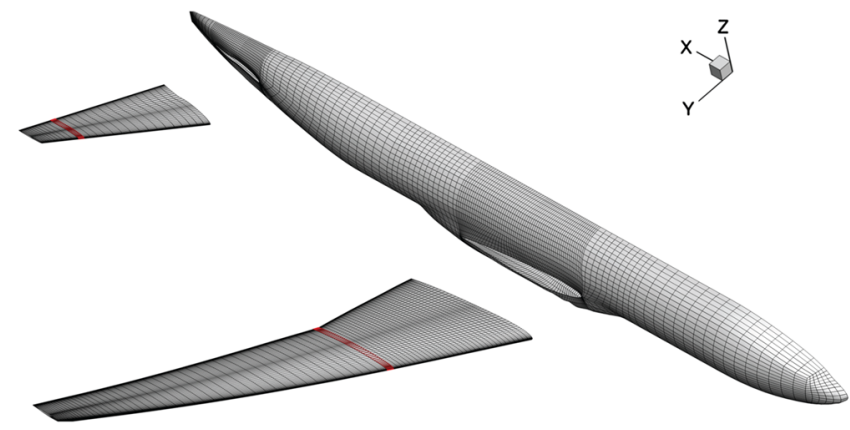

Fig. 1 Three separate meshes used for the viscous drag computation. Representative strips are shown for the wing and tail grids. 
Substituting the solution of Eq. (ㅁ) into Eq. (4) to eliminate the total derivatives, we obtain

$$
\frac{\mathrm{d} I}{\mathrm{~d} x}=\frac{\partial I}{\partial x}-\underbrace{\left[\begin{array}{ll}
\frac{\partial I}{\partial w} & \frac{\partial I}{\partial u}
\end{array}\right]\left[\begin{array}{ll}
\frac{\partial \mathcal{A}}{\partial w} & \frac{\partial \mathcal{A}}{\partial u} \\
\frac{\partial \mathcal{S}}{\partial w} & \frac{\partial \mathcal{S}}{\partial u}
\end{array}\right]^{-1}}_{\Psi^{T}}\left[\begin{array}{l}
\frac{\partial \mathcal{A}}{\partial x} \\
\frac{\partial \mathcal{S}}{\partial x}
\end{array}\right]
$$

There are two techniques for solving this equation. The first is to solve Eq. (5) for $\mathrm{d} w / \mathrm{d} x$ and $\mathrm{d} u / \mathrm{d} x$ once for design variable $x$. This technique is known as the direct method. The second approach is the adjoint method. In the adjoint method, we solve for the coupled adjoint vectors $\psi$ and $\phi$, once for each function of interest, using the coupled adjoint equations:

$$
\left[\begin{array}{ll}
\frac{\partial \mathcal{A}}{\partial w} & \frac{\partial \mathcal{A}}{\partial u} \\
\frac{\partial \mathcal{S}}{\partial w} & \frac{\partial \mathcal{S}}{\partial u}
\end{array}\right]^{T}\left[\begin{array}{l}
\psi \\
\phi
\end{array}\right]=\left[\begin{array}{ll}
\frac{\partial I}{\partial w} & \frac{\partial I}{\partial u}
\end{array}\right]^{T}
$$

If there are more design variables than functions of interest, as is the case for our optimization problems, it is more computationally efficient to use the adjoint method. Once the adjoint vector for a function of interest has been found, the total sensitivity can be determined by rearranging Eq. (6):

$$
\frac{\mathrm{d} I}{\mathrm{~d} x}=\frac{\partial I}{\partial x}-\psi^{T}\left(\frac{\partial \mathcal{A}}{\partial x}\right)-\phi^{T}\left(\frac{\partial \mathcal{S}}{\partial x}\right)
$$

The coupled adjoint equations (7) can be solved using either a segregated Gauss-Seidel approach, as was used for the results in Sec. IV, or directly using a Krylov approach. All of the partial derivative terms, the mesh movement, the coupling procedure, and the solution procedures are computed in a fully parallel fashion to ensure that the entire sensitivity analysis does not suffer from serial bottlenecks. The computational costs of the setup and solution for the coupled adjoint method are approximately 35 and 60\%, respectively, of the cost of an aerostructural solution. More details on the verification and computational performance of the coupled adjoint implementation can be found in Kenway et al. [35].

\section{Problem Description}

The baseline geometry for the optimization is the NASA Common Research Model (CRM) [51] wing-body-tail configuration, which is publicly available and exhibits the design features typical of a transonic, wide-body, long-range aircraft. The configuration has been carefully designed to yield good aerodynamic performance across a range of Mach numbers and lift coefficients, providing a reasonable starting aerodynamic shape for the MDO.

Because the CRM geometry defines only the outer mold line, we create a structural model that conforms to the wing outer mold line and is representative of a modern airliner structural wing box. The CRM outer mold line is a $1 g$ flying shape. Because the original jig shape is not available, we designate the supplied shape as the jig shape for the purposes of our optimizations. As a result, the wing shape generated for a $1 g$ condition is not the same as the CRM configuration. The structural model contains 38 ribs; two spars at approximately 10 and $75 \%$ of the local chord; 20 stringers from the root to the Yehudi break and 11 stringers from the Yehudi break to the tip; and vertical rib stiffeners. We consider only the structural model of the wing because this is where the aerostructural coupling is the most critical for the design. The CFD surface mesh and the structural model are shown in Fig. 2.

In addition to the outer mold line and the wing structural model, we require some further information to formulate the aircraft design optimization problem. Specifically, we need to know the design

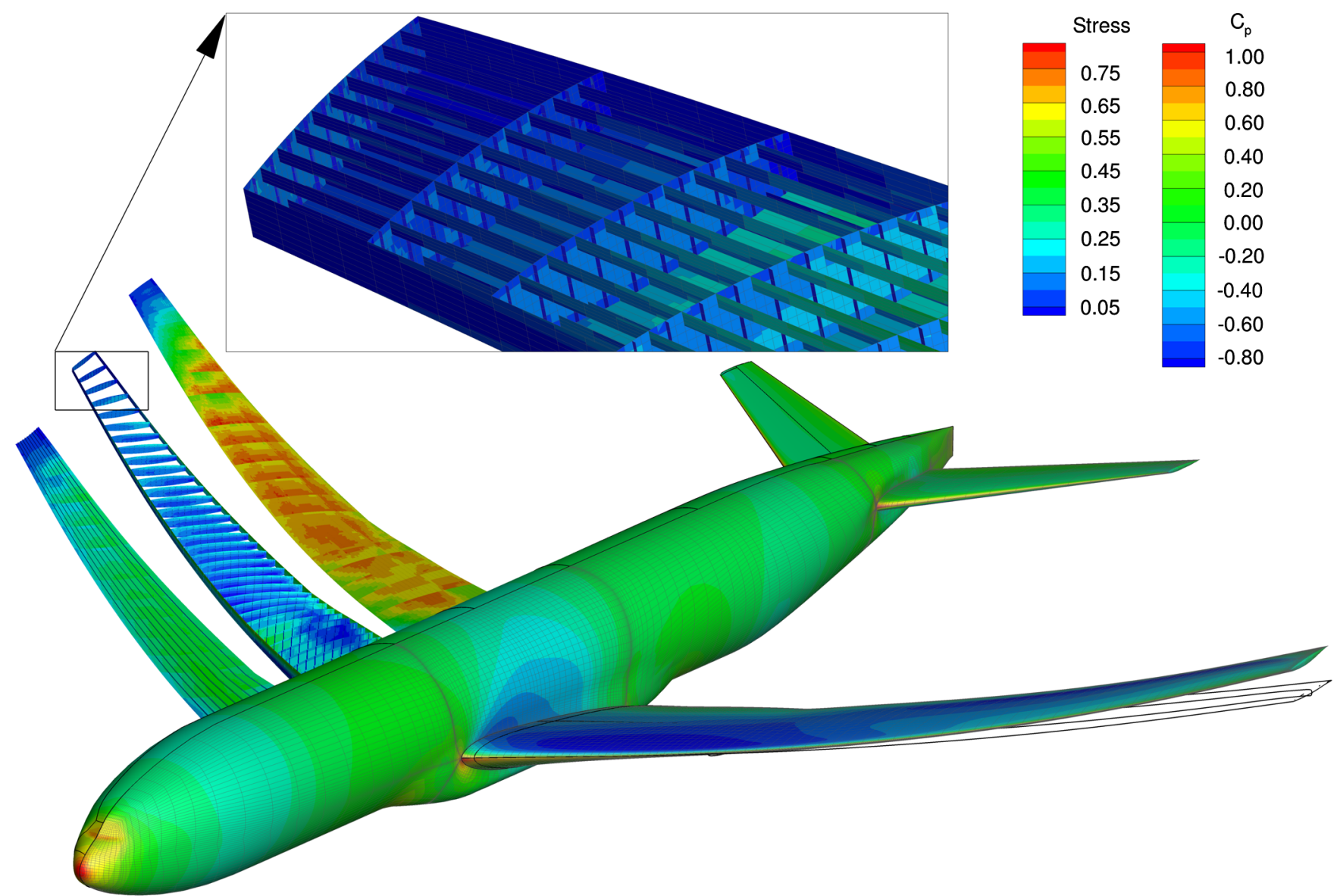

Fig. 2 Common research model aerostructural solution at Mach 0.85; CFD solution at $1 g$ shown on the right, and structural model and solution at $2.5 g$ shown on the left. 
Table 1 CRM specifications

\begin{tabular}{lc}
\hline \hline Parameter & Value \\
\hline Cruise Mach number & 0.85 \\
Cruise lift coefficient & 0.5 \\
Initial cruise altitude & $35,000 \mathrm{ft}$ \\
Span & $58.6 \mathrm{~m}$ \\
Aspect ratio & 9.0 \\
Reference wing area & $383.7 \mathrm{~m}^{2}$ \\
Sweep (leading edge) & $37.4 \mathrm{deg}$ \\
MTOW & $298,000 \mathrm{~kg}$ \\
Operational empty weight & $138,100 \mathrm{~kg}$ \\
Design range & $7,725 \mathrm{~mm}$ \\
Design payload & $34,000 \mathrm{~kg}$ \\
Reserve fuel & $15,000 \mathrm{~kg}$ \\
Initial wing weight & $29,200 \mathrm{~kg}$ \\
Secondary wing weight & $8,000 \mathrm{~kg}$ \\
Fixed weight & $100,900 \mathrm{~kg}$ \\
Thrust-specific fuel consumption $(c)$ & $0.53 \mathrm{lb} /(\mathrm{lbf} \cdot \mathrm{h})$ \\
\hline \hline
\end{tabular}

mission range and payload, as well as the operational empty weight and maximum takeoff weight (MTOW). Because the overall dimensions of the CRM are very similar to those of the Boeing 777200ER, we take this information from the publicly available documentation [52]. Table $\underline{1}$ lists the key parameters used for the optimizations.

\section{A. Objective Function}

The choice of objective function has a significant impact on the optimized aerostructural design. The objective function must be carefully chosen to accurately reflect the design intent for a particular aircraft. In many ways, this is a much more challenging problem for aerostructural analysis than it is for separate aerodynamic and structural analyses. As an example, consider a common multidisciplinary objective, the aircraft range as given by the Breguet equation:

$$
R=\frac{V}{c_{T}} \frac{C_{L}}{C_{D}} \ln \left(\frac{W_{1}}{W_{2}}\right)
$$

where $R$ is the range, $V$ is the flight speed, $c_{T}$ is the thrust-specific fuel consumption, and $W_{1}$ and $W_{2}$ are the initial and final cruise weights, respectively. If the range equation is used as an objective for aerodynamic optimization, the ratio of the cruise weights remains fixed, and given a constant Mach number and thrust-specific fuel consumption, maximizing the range reduces to a maximization of $L / D$ or a lift-constrained drag minimization. This simplification ignores the multidisciplinary effects of the aircraft design range. Similarly, if we consider only a structural optimization, then the only relevant metric is the weight ratio, and the problem becomes a failureconstrained weight-minimization problem.

In both cases, the single-discipline optimization problems lead to simplified formulations that ignore the terms that are assumed constant. An aerostructural optimization can account for the $L / D$ and ln $\left(W_{1} / W_{2}\right)$ terms simultaneously, and it is critical that the tradeoff between these terms accurately reflects the intent of the designer.

A quantitative analysis can be performed to determine the tradeoff between drag and weight reduction that answers the question, "How many kilograms of structural mass is equivalent to one drag count for a given objective function?" We start by linearizing the Breguet range equation (9) with respect to the drag coefficient and the final cruise weight:

$$
R\left(C_{D}, W_{2}\right)=\frac{\partial R}{\partial C_{D}} C_{D}+\frac{\partial R}{\partial W_{2}} W_{2}=\gamma C_{D}+\beta W_{2}
$$

Here, the partial derivatives are computed at the reference values $C_{D_{0}}$ and $W_{2_{0}}$ to yield a linearization about those values:

$$
\gamma=\frac{\partial R}{\partial C_{D}}=-\frac{V}{c_{T}} \frac{C_{L}}{C_{D_{0}}^{2}} \ln \left(\frac{W_{1}}{W_{2_{0}}}\right)
$$

$$
\beta=\frac{\partial R}{\partial W_{2}}=-\frac{V}{c_{T}} \frac{C_{L}}{C_{D_{0}}} \frac{1}{W_{2_{0}}}
$$

The ratio of these partial derivatives is

$$
\frac{\gamma}{\beta}=\frac{\partial W_{2}}{\partial C_{D}}=\frac{W_{2_{0}}}{C_{D_{0}}} \ln \left(\frac{W_{1}}{W_{2_{0}}}\right)
$$

The ratio $\gamma / \beta$ corresponds to the partial derivative $\partial W_{2} / \partial C_{D}$, which quantifies the decrease in the final cruise weight that would increase the range by the same amount as a unit decrease in the drag coefficient. Thus, the ratio $\gamma / \beta$ answers the previous question: it quantifies how many units of structural mass are equivalent to a unit of drag. In this case, we derived this quantity for range, but we will also derive it for TOGW and fuel burn.

The partial derivative given by Eq. (13) shows us that the weightdrag tradeoff scales with the final cruise weight, the natural logarithm of the weight ratio, and that it is inversely proportional to the cruise drag coefficient. Unlike the single-discipline optimization cases, where a simple reduction of drag or weight was sufficient, this multidisciplinary objective requires knowledge of the aerodynamic performance $C_{D}$ and the structural performance, $\ln \left(W_{1} / W_{2}\right)$, as well as their coupling, to determine the correct multidisciplinary trades. This places additional burden on the analysis because we must include all of the components of the drag and weight to achieve the correct aerostructural tradeoffs, even if they are not modeled explicitly.

In this work, we consider the minimization of two objectives: TOGW and fuel burn. The TOGW objective combines the manufacturing cost (which depends on the empty weight) and the fuel burn. The fuel burn accounts for a large portion of the direct operating cost (DOC), especially for long-range commercial aircraft. The real objective function for commercial aircraft is usually a compromise between these two objectives.

The TOGW is assumed to be equal to the initial cruise weight because we consider the fuel burn only during the cruise segment and ignore the fuel burned during takeoff, climb, and descent. Rearranging the Breguet equation (9), we can obtain an expression for the TOGW, given a fixed range:

$$
\mathrm{TOGW}=W_{1}=W_{2} e^{\left(R c_{T} / V\right)\left(C_{D} / C_{L}\right)}
$$

Linearizing this objective function with respect to $C_{D}$ and $W_{2}$ about the reference point $\left(C_{D_{0}}, W_{2_{0}}\right)$, we obtain

$$
\begin{gathered}
\gamma=e^{R c_{T} C_{D_{0}} / V C_{L}} W_{2_{0}}\left(\frac{R c_{T}}{V C_{L}}\right)=\frac{W_{1}}{C_{D_{0}}} \ln \left(\frac{W_{1}}{W_{2_{0}}}\right) \\
\beta=e^{R c_{T} C_{D_{0}} / V C_{L}}=\frac{W_{1}}{W_{2_{0}}}
\end{gathered}
$$

The ratio of these two sensitivities is

$$
\frac{\gamma}{\beta}=\frac{W_{2_{0}}}{C_{D_{0}}} \ln \left(\frac{W_{1}}{W_{2_{0}}}\right)
$$

An equation for the cruise-segment fuel burn can also be obtained from the Breguet equation (9) as follows:

$$
W_{f}=W_{1}-W_{2}=W_{2}\left(e^{\left(R c_{T} / V\right)\left(C_{D} / C_{L}\right)}-1\right)
$$


Table 2 Linearization of the objectives and the values of the corresponding sensitivities for the CRM configuration

\begin{tabular}{lcccccc}
\hline \hline Objective, $I$ & $\gamma=\partial I / \partial C_{D}$ & $\begin{array}{c}\text { Kilograms } \\
\text { per count }\end{array}$ & $\beta=\partial I / \partial W_{2}$ & $\begin{array}{c}\text { Kilograms per } \\
\text { kilogram }\end{array}$ & $\begin{array}{c}\text { Kilograms } \\
\text { per count }\end{array}$ \\
\hline TOGW & $W_{1} / C_{D_{0}} \ln \left(W_{1} / W_{2_{0}}\right)$ & 497.0 & $W_{1} / W_{2_{0}}$ & 1.59 & $W_{2_{0} / C_{D_{0}} \ln \left(W_{1} / W_{2_{0}}\right)}$ \\
Fuel burn & $W_{1} / C_{D_{0}} \ln \left(W_{1} / W_{2_{0}}\right)$ & 497.0 & $\left(W_{1}-W_{2_{0}}\right) / W_{2_{0}}$ & 0.59 & $W_{1} W_{2_{0}} / C_{D_{0}}\left(W_{1}-W_{2_{0}}\right) \ln \left(W_{1} / W_{2_{0}}\right)$ & 842.4 \\
\hline \hline
\end{tabular}

$$
\begin{gathered}
\gamma=e^{R c_{T} C_{D_{0}} / V C_{L}} W_{2_{0}}\left(\frac{R c_{T}}{V C_{L}}\right)=\frac{W_{1}}{C_{D_{0}}} \ln \left(\frac{W_{1}}{W_{2_{0}}}\right) \\
\beta=e^{R c_{T} C_{D_{0}} / V C_{L}}-1=\frac{W_{1}}{W_{2_{0}}}-1
\end{gathered}
$$

The ratio of these two sensitivities is

$$
\frac{\gamma}{\beta}=\frac{W_{1} W_{2_{0}}}{C_{D_{0}}\left(W_{1}-W_{2_{0}}\right)} \ln \left(\frac{W_{1}}{W_{2_{0}}}\right)
$$

These partial derivatives and their ratios are summarized in Table 2. In addition, we calculated the values of these quantities for the CRM specifications listed in Table 1 .

The resulting $\gamma / \beta$ for the TŌGW objective is identical to that of the range objective. Therefore, in the context of this linearization, maximizing the range for a fixed TOGW is equivalent to minimizing the TOGW for a fixed range. However, in their nonlinear form, these objectives are not equivalent because changing the range or TOGW will modify the resulting linearization.

The TOGW objective $\gamma / \beta$ differs from the fuel burn $\gamma / \beta$ by a factor of $\left(W_{1}-W_{2}\right) / W_{1}=W_{f} / W_{1}$, which is the fuel fraction. Because the fuel fraction is always less than 1 , the $\gamma / \beta$ ratio for the fuel-burn objective is always higher than the one for the TOGW objective. A higher $\gamma / \beta$ indicates that one drag count is equivalent to a larger mass. Thus, a higher $\gamma / \beta$ value favors designs that have better aerodynamic performance, where the weight is more readily increased to reduce the drag. Lower $\gamma / \beta$ values, on the other hand, favor structural weight reduction over drag reduction.

The fuel fraction for the CRM aircraft for the design payload and the range is $\left(W_{1}-W_{2}\right) / W_{1}=0.372$, and so the fuel burn objective $\gamma / \beta$ is 2.69 times larger than the TOGW one. For the TOGW objective, one drag count is worth approximately $313 \mathrm{~kg}$, but for the fuel-burn objective, one drag count is equivalent to $842 \mathrm{~kg}$. These values, however, are based on linearizations, and so they do not take into account that a reduction in $W_{2}$ further decreases the TOGW and fuel burn when the weight calculation is recomputed. This analysis assumes a design range of $7725 \mathrm{~nm}$. Figure $\underline{3}$ shows the numerical value of the weight-drag tradeoff as a function of the range. The $\gamma / \beta$ increases with respect to the range for both objectives, indicating that longer-range aircraft should place greater emphasis on the aerodynamic performance. As the range decreases, so does the fuel fraction, and the ratio of $\gamma / \beta$ for the two objectives grows without bounds as the range approaches zero. This dependence of $\gamma / \beta$ on the range can only be captured with a multidisciplinary analysis.

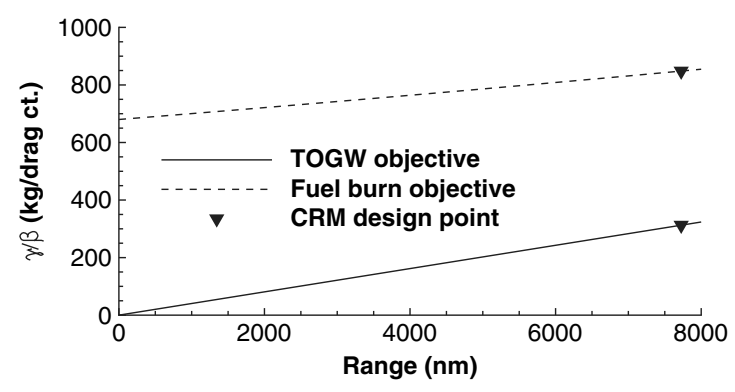

Fig. 3 Variation of TOGW and fuel-burn objective sensitivities as a function of design range.
As discussed previously, $\gamma / \beta$ is much higher for the fuel-burn objective than for the TOGW objective. Therefore, minimizing the former objective places a greater emphasis on decreasing drag, and the emphasis increases with the range for both objectives, as shown in Fig. 3 . We can also observe that $\gamma / \beta$ increases more rapidly with the range for the TOGW objective.

For the optimizations presented in Sec. IV, we compute the final cruise weight $W_{2}$ according to

$$
\begin{gathered}
W_{2}=W+\text { Fixed Weight }+ \text { Reserve Fuel Weight } \\
+A / A_{\text {ref }} \times \text { Secondary Wing Weight }
\end{gathered}
$$

where $W$ is the weight of the primary wing structure given based on the volume of the structural finite-element model, $A$ is the projected wing area, and $A_{\text {ref }}$ is the initial projected wing area. The last term is used to account for the weight of fasteners and other wing parts that scale with the wing area. For each of the cruise conditions described in the following section, the $L / D$ ratio and the Breguet range equation are used to determine the weight at the start of cruise, $W_{1}$. The $L / D$ ratio for each condition is computed by performing an aerostructural analysis at the weight corresponding to the midpoint of the mission. Finally, for each of the objective functions considered, we use the average over each cruise condition to formulate the composite objectives.

\section{B. Design and Maneuver Conditions}

We now describe the operating conditions used in the optimizations. To evaluate the TOGW and fuel-burn objectives, we estimate the weight at the beginning of cruise using the Breguet range equation:

$$
R=\frac{V}{c_{T}} \frac{C_{L}}{C_{D}} \ln \left(\frac{W_{1}}{W_{2}}\right)
$$

We assume that the aircraft is able to climb continuously as fuel is consumed, while maintaining the same lift-to-drag ratio and cruise Mach number. The only aerodynamic input to this computation is the overall lift-to-drag ratio of the aircraft.

Although a single analysis point is sufficient to estimate the lift-todrag ratio, single-point optimizations (especially in the transonic regime) can produce optimal performance at a single operating point at the cost of significant degradation in other important off-design conditions [53]. To address this issue, we consider multipoint aerostructural optimizations that compute the average performance over multiple flight conditions. We consider the five cruise operating conditions listed in Table $\underline{3}$ and labeled $\mathrm{C} 1$ through $\mathrm{C} 5$. These points form a cross in the Mach-altitude space centered about the operating design condition for the CRM configuration $\left(M=0.85, C_{L}=0.5\right)$, as shown in Fig. 4. The cruise Mach number is varied by \pm 0.01 , and the altitude is varied by $\pm 1000 \mathrm{ft}$. The change in altitude effectively varies $C_{L}$ to represent typical in-service variations for a given mission. The design is particularly sensitive to the cruise Mach number because of the nonlinear nature of the compressible flow and because the optimizer can usually eliminate wave drag for a single Mach number.

Two separate maneuver conditions, labeled M1 and M2 in Table $\underline{3}$, are considered: a $2.5 g$ symmetric pull-up maneuver, and a $1 . \overline{3} g$ acceleration due to gust. M1 represents a $2.5 \mathrm{~g}$ limit load for the wing structure. M2 is meant to emulate the unsteady gust loads that, in practice, limit the lower-wing skin and stringers because of a fatigue 
Table 3 Operating conditions

\begin{tabular}{lcccc}
\hline \hline Group & Identifier & Mach & Altitude, ft & Load factor \\
\hline Cruise & C1 & 0.85 & 35,000 & 1.0 \\
Cruise & C2 & 0.84 & 35,000 & 1.0 \\
Cruise & C 3 & 0.86 & 35,000 & 1.0 \\
Cruise & C4 & 0.85 & 34,000 & 1.0 \\
Cruise & C5 & 0.85 & 36,000 & 1.0 \\
Maneuver & M1 & 0.86 & 20,000 & 2.5 \\
Maneuver & M2 & 0.85 & 32,000 & 1.3 \\
Stability & S1 & 0.85 & 35,000 & 1.0 \\
\hline \hline
\end{tabular}

life limit [54]. We implement this constraint by performing a static aeroelastic analysis at $1.3 \mathrm{~g}$ and then limiting the stress at this condition to a value that is significantly lower than the yield stress, which is determined based on the fatigue life, as described in Sec. III.D.

A last aerostructural analysis, labeled $\mathrm{S} 1$ in Table 3 , is required to estimate the aircraft's static margin. Using static aeroelastic analysis, we estimate the static margin of the deformed configuration using the following formula:

$$
K_{n}=-\frac{C_{M_{\alpha}}}{C_{L_{\alpha}}}
$$

Further details on approximating static and dynamic stability derivatives for high-fidelity CFD optimization can be found in Mader and Martins [18]. The derivatives $C_{M_{\alpha}}=\partial C_{M} / \partial \alpha$ and $C_{L_{\alpha}}=$ $\partial C_{L} / \partial \alpha$ are estimated using a forward finite difference with step size of $\Delta \alpha=0.1 \mathrm{deg}$. Because these coefficients are nearly linear in the range under consideration, we can use this relatively large finite difference step without significant truncation error, while avoiding the subtractive cancellation errors that would show up for smaller steps. The analysis for cruise condition $\mathrm{C} 1$ is used for the baseline value, and the additional stability point provides the perturbed value to complete the derivative calculations.

\section{Design Variables}

The two optimizations presented use hundreds of design variables to parametrize the aerodynamic and structural models. As is typical in a multidisciplinary analysis, we can divide the design variables into global variables, which directly affect more than one discipline, and local variables, which affect only a single discipline. Table $\underline{4}$ lists all of the optimization variables.

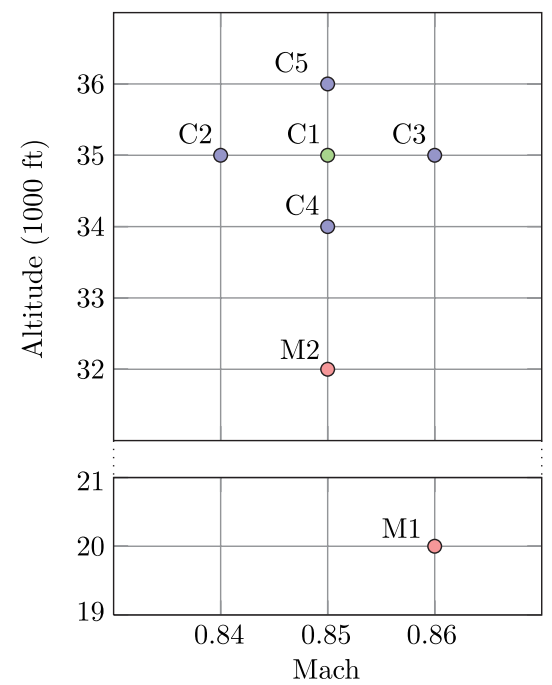

Fig. 4 Cruise and maneuver conditions in Mach-altitude space.
Table 4 Design variables

\begin{tabular}{|c|c|}
\hline Description & Quantit \\
\hline \multicolumn{2}{|c|}{ Global variables } \\
\hline Span & 1 \\
\hline Sweep & 2 \\
\hline Chord & 4 \\
\hline Twist & 5 \\
\hline Shape & 160 \\
\hline \multicolumn{2}{|c|}{ Aerodynamic variables } \\
\hline Angle of attack & 1 \\
\hline Tail rotation & 1 \\
\hline \multicolumn{2}{|c|}{ Structural variables } \\
\hline Upper skin & 54 \\
\hline Lower skin & 54 \\
\hline Upper stringers & 54 \\
\hline Lower stringers & 54 \\
\hline Ribs & 18 \\
\hline Rib stiffeners & 18 \\
\hline Spars & 36 \\
\hline Total & 472 \\
\hline
\end{tabular}

We use a free-form deformation (FFD) volume approach to make geometric perturbations to the geometry. Further information on our approach can be found in Kenway et al. [55]. The main wing planform variables (span, sweep, chord, and twist) are all global variables because they directly affect the geometry of both the aerodynamics and the structures. The geometric and structural design variables and the CFD mesh discretrization used for the optimization are shown in Fig. 5. The chords are modified at the root, Yehudi break, near-tip, and tip sections, and the remaining sections are linearly interpolated. Five twist angles are defined similarly and interpolated linearly in the spanwise direction. Two sweep variables are specified. The first sweeps nearly the entire leading edge, which (by construction) is constrained to remain straight. The second changes only the outer $12.5 \%$ of the wing semispan. The shape variables are used to perturb the coefficients of the FFD volume surrounding the wing in the $z$ (normal) direction. These shape variables prescribe the chord and spanwise airfoil shape directly, and no additional explicit thickness or camber variables are required. Figure $\underline{5}$ shows the internal layout of the structure as well as the grouping of the structural design variables.

The structural skin-thickness variables are grouped in a grid of 18 stations in the spanwise direction and three stations in the chordwise directions, resulting in 54 variables for each of the upper and lower skins. The stringer variables are grouped in the same way. The ribs and rib stiffeners each have 18 variables in the spanwise direction, as do each of the leading- and trailing-edge spars. The stringer and rib pitches are not fixed and vary with the planform variables. Each of the five cruise conditions and two maneuver conditions have an independent angle of attack and tail rotation angle to provide the required degrees of freedom to meet both the lift and moment constraints. Finally, the mean aerodynamic chord (MAC) and center of gravity location $\left(X_{\mathrm{CG}}\right)$ are target variables, each coupled with a consistency constraint that simplifies the implementation. This represents an interdisciplinary feasible MDO approach applied to these coupling variables [3]. The initial values for the geometric and aerodynamic design variables are chosen to reproduce the original CRM geometry exactly.

To establish a reasonable initial structural design for the aerostructural optimization, we optimize the wing box by minimizing the structural weight with respect to the structural thicknesses described previously, subject to stress constraints for a set of fixed aerodynamic loads. The fixed loads are obtained from aerostructural analyses at the two maneuver conditions described previously. The initial design consists of a structure with linear spanwise thickness variation in each of the structural members. The stress constraints are identical to those described next, in Sec. III.D. By using design variables and constraints consistent with those used in the aerostructural optimizations, we provide an initial wing box that has already 


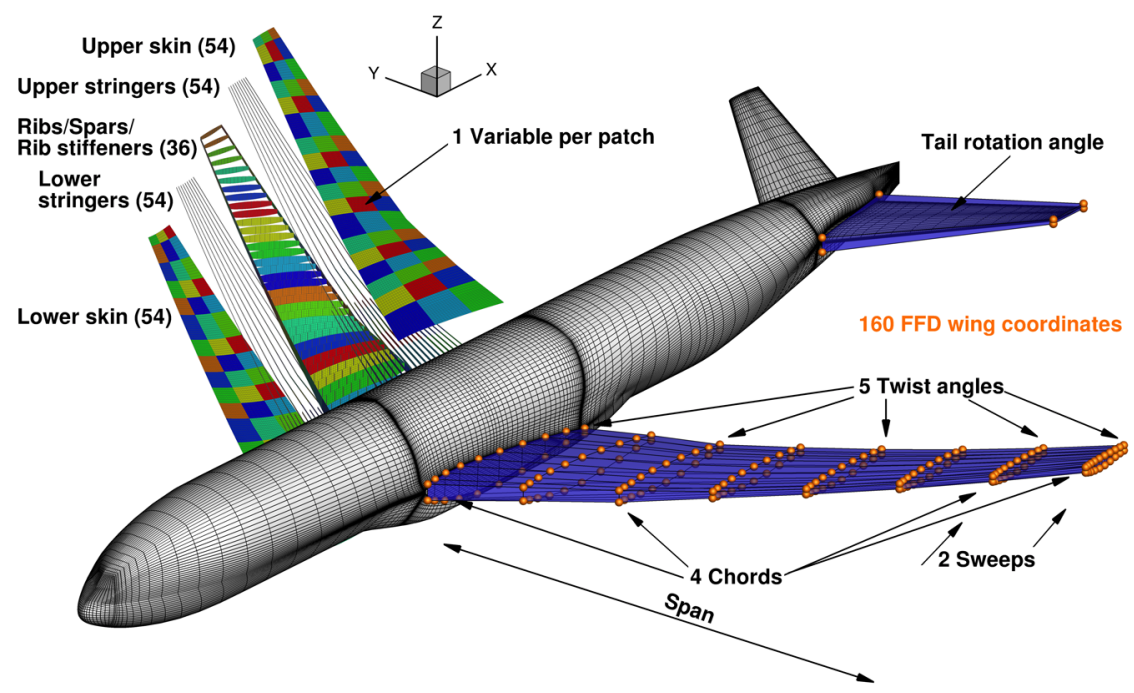

Fig. 5 Optimization design variables: structural design variable grouping (left), and geometric design variables (right).

been optimized in a subspace of the aerostructural optimizations, and subsequent improvements in the full space demonstrate the importance of considering both disciplines. The initial structure resulting from this optimization is shown in Sec. IV.

\section{Design Constraints}

In this section, we describe the constraints that are used for the TOGW and fuel-burn optimizations. For simplicity, we divide the constraints into three groups: geometric and target constraints, aerodynamic constraints, and structural constraints, as shown in Table 5. The first two geometric constraints, $t_{\mathrm{LE}}$ and $t_{\mathrm{TE}}$, are used to constrain the initial wing thickness at 2.5 and $97.5 \%$ of the chord of each airfoil. The thickness constraints at $2.5 \%$ chord constrain the leading-edge radius and help to ensure that the high-speed aerostructural optimization does not significantly impact the lowspeed $C_{L_{m}}$ performance, which is largely governed by the roundness of the leading edge. The thickness constraints at $97.5 \%$ chord prevent the upper and lower surfaces from crossover near the sharp trailing edge. The projected wing area is constrained to be no less than the initial area. This constraint is used to ensure adequate takeoff and landing field length performance. Even with a complete structural model, a minimum fuel-volume constraint is required. Only the

\section{Table 5 Optimization constraints}

\begin{tabular}{|c|c|}
\hline Description & Quantity \\
\hline \multicolumn{2}{|c|}{ Geometric/target constraints } \\
\hline$t_{\mathrm{LE}} / t_{\mathrm{LE}_{\text {Init }}} \geq 1.0$ & 11 \\
\hline$t_{\mathrm{TE}} / t_{\mathrm{TE}_{\text {Init }}} \geq 1.0$ & 11 \\
\hline$A / A_{\text {init }} \geq 1.0$ & 1 \\
\hline$V / V_{\text {init }} \geq 1.0$ & 1 \\
\hline$t_{\text {TESpar }} \geq 0.20$ & 5 \\
\hline$t_{\text {Tip }} / t_{\text {Tip }} \geq 0.5$ & 5 \\
\hline $\mathrm{MAC}-\mathrm{MAC}^{*}=0.0$ & 1 \\
\hline$X_{\mathrm{CG}}-X_{\mathrm{CG}}^{*}=0.0$ & 1 \\
\hline \multicolumn{2}{|c|}{ Aerodynamic constraints } \\
\hline Cruise: $L-W=0.0$ & 5 \\
\hline Cruise: $C_{m_{v}}=0.0$ & 5 \\
\hline Maneuver: $L-W=0.0$ & 2 \\
\hline Maneuver: $C_{m_{y}}=0.0$ & 2 \\
\hline Static margin: $K_{n} \geq 0.15$ & 1 \\
\hline \multicolumn{2}{|c|}{ Structural constraints } \\
\hline $2.5 g$ lower skin: $K S \leq 1.0$ & 1 \\
\hline $2.5 g$ upper skin: $K S \leq 1.0$ & 1 \\
\hline $2.5 \mathrm{~g}$ rib/spars: $K S \leq 1.0$ & 1 \\
\hline $1.3 g$ lower skin: $K S \leq 0.42$ & 1 \\
\hline $1.3 \mathrm{~g}$ upper skin: $K S \leq 1.0$ & 1 \\
\hline $1.3 g$ rib/spars: $K S \leq 1.0$ & 1 \\
\hline Total & 57 \\
\hline
\end{tabular}

volume inside the spar box is computed to ensure that the optimized wing is able to carry at least the same amount of fuel as the initial design.

Several additional thickness constraints are also enforced. Minimum trailing-edge spar height constraints $t_{\text {TEspar }}$ are used over the outboard section of the wing. These constraints are intended to ensure that adequate vertical space is available to attach the actuation devices required for the flaps and ailerons. The tip thickness constraint $t_{\text {tip }}$ is used to ensure that the optimization does not produce an unrealistically thin wing tip.

Each cruise and maneuver condition enforces equality constraints on the lift and pitching moment coefficient, so that all of the aerostructural solutions are trimmed. The static margin of the $\mathrm{C} 1$ condition is constrained to be greater than $15 \%$. The reference point for the moment computation is taken to be at $25 \%$ of MAC, which changes with the planform design variables. By including a pitch moment constraint as well as a constraint on the full configuration static margin, we allow the optimization to trade drag reduction from aft-loaded supercritical profiles with the induced drag penalty required to trim the configuration.

Last, we must constrain the stresses on the structure. The structural model used for the optimization consists of over 50,000 secondorder MITC shell elements [56]. Individually constraining the stress in each of these elements would require the solution of the corresponding number of coupled adjoint vectors, which would incur a prohibitive computational cost. To reduce the number of solutions required, we use the KS constraint aggregation technique [50,57] that conservatively estimates the maximum of a set of stresses in a smooth and differentiable manner. Each maneuver condition uses three KS functions: the first for the lower-wing skin and stringers, the second for the upper-wing skin and stringers, and the third for the spars, ribs, and rib stiffeners. The compression members in the upper-wing skin and stringers are assumed to be Aluminum 7050 with a maximum allowable stress of $300 \mathrm{MPa}$. The remainder of the primary wing structure is assumed to be manufactured from Aluminum 2024 with a maximum allowable stress of $324 \mathrm{MPa}$.

For the first maneuver condition, the maximum von Mises stress must be below the limiting stress, which requires the three KS functions to be less than 1. For the second maneuver constraint, which is related to fatigue, we limit the stress on the lower-wing skin and stringers to be below $138 \mathrm{MPa}$ for the $1.3 \mathrm{~g}$ load condition, corresponding to an upper limit of 0.42 [54]. The remaining two KS functions for the $1.3 \mathrm{~g}$ maneuver condition retain the maximum $\mathrm{KS}$ value of 1 .

\section{E. Optimization Algorithm}

For design optimization problems with hundreds of design variables (476 in our case), and objective and constraint evaluations 
requiring on the order of several minutes, a gradient-based optimization algorithm is the only viable option to get an accurate answer in under two days. For this work, we use SNOPT [58] through the pyOpt interface [59]. SNOPT is well-suited for large-scale constrained nonlinear optimization problems. The combination of the coupled adjoint, which can compute the gradient of a function of interest in approximately the same time as a function evaluation, with effective gradient-based optimization enables us to solve each optimization problem in approximately $36 \mathrm{~h}$.

\section{F. Computational Resources}

The two optimizations are performed on a massively parallel supercomputer [60]. Each optimization function evaluation requires the solution of eight aerostructural solutions: five for the cruise conditions, two for the maneuver conditions, and one for the stability point. To reduce the wall time required for the optimizations, these parallel analyses are carried out concurrently. The cruise conditions and stability condition use the 2.1 million cell CFD mesh with 52 processors and four processors for the CFD and CSM problems, respectively. The two maneuver conditions use a smaller CFD mesh with 1.2 million cells and the same structural discretrization. A smaller grid is used for the maneuver conditions because accurate drag predictions are not required for these cases. The maneuver conditions use 45 CFD processors and four CSM processors. To ensure good overall computational efficiency for the optimization problem, we try to balance the time required for each concurrent analysis; these times should be approximately equal to avoid idle processes. Load balancing is complicated by the fact that the cruise analyses require the computation of three adjoint vectors (lift, drag, and moment), the maneuver conditions require five adjoint vectors (lift, moment, and three KS functions), and the stability condition requires only two (lift and moment). The optimization algorithm itself is serial and does not contribute significantly to the overall computational cost. The number of processors used for each aerostructural computation is shown in Fig. 6 . The total number of processors used for each optimization is $(56 \times 5)+(49 \times 2)+$ $56+1=435$. The single additional processor at the end is used for the viscous-drag computation. The TOGW optimization required $34.5 \mathrm{~h}$ of wall time, while the fuel-burn optimization required $36.5 \mathrm{~h}$.

\section{Results}

We now examine the results from two optimization runs: a TOGW minimization and a fuel-burn minimization. The results are presented concurrently to compare the effects of choosing a different objective function.

First, we examine the optimization convergence history for each problem, which is shown in Fig. 7. Feasibility is the maximum constraint violation, and it is a measure of how closely the nonlinear constraints are satisfied. Optimality refers to how closely the current point satisfies the first-order Karush-Kuhn-Tucker conditions [58]. Additionally, the evolution of each objective is shown. The optimizer performed 150 major optimization iterations to reduce the optimality criterion by two orders of magnitude. We have found that these complex aerostructural optimization problems converge slowly. However, the majority of the objective improvement is accomplished in our optimizations, and significant additional computational effort is required to reduce the objective further. The optimizations are
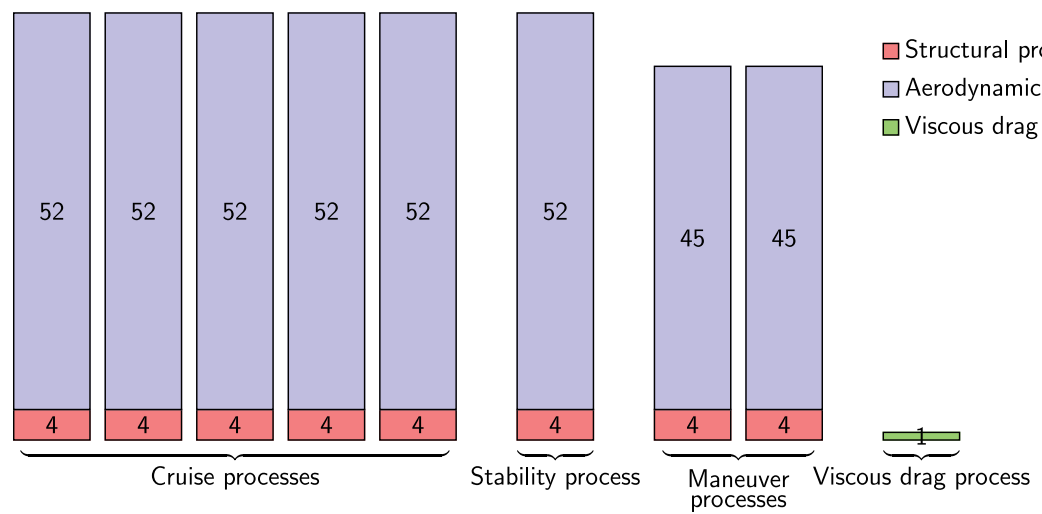

Fig. 6 Processor groups for the multipoint aerostructural optimization.
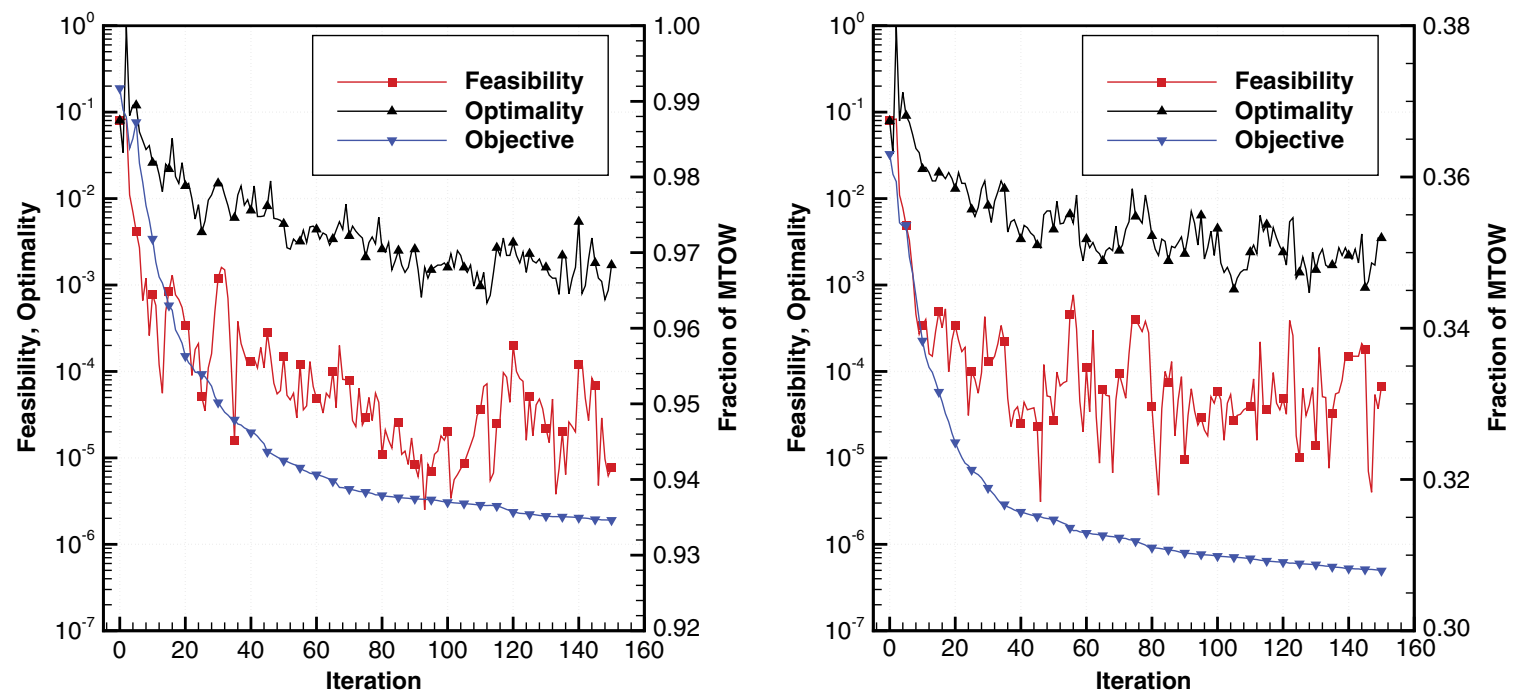

a) TOGW optimization

b) Fuel-burn optimization

Fig. 7 Optimization convergence histories for each optimization. 


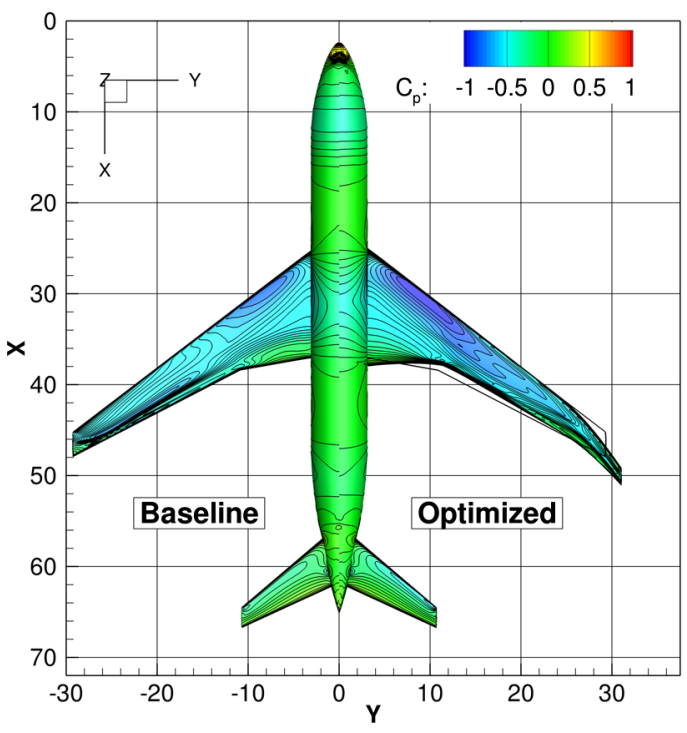

a) TOGW optimization

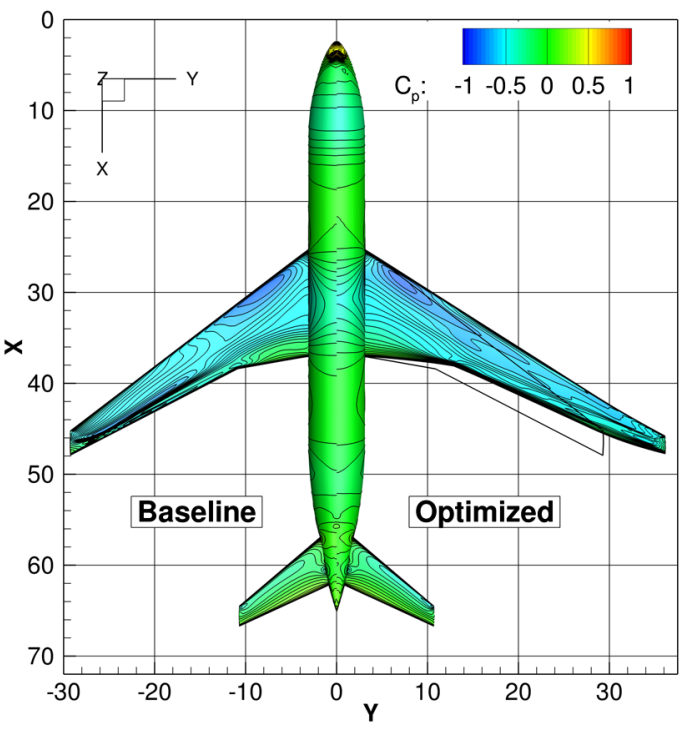

b) Fuel-burn optimization

Fig. 8 Planform view with $C_{p}$ contours for both optimum designs for $\mathrm{C} 1$ condition. The baseline design is included for reference.

sufficiently converged to allow us to draw overall conclusions and to examine the design tradeoffs in the resulting optimal design.

Now we compare the wing planform changes in the optimized designs. The chord, sweep, and span variables (seven in total) control the planform, including the wing area. Figure $\underline{8}$ compares the new planforms with the original design. Despite the large amount of design flexibility, the optimized designs have reasonable planforms, which indicates that the integration of the structural design and constraints successfully prevented the aerodynamic shape optimization from obtaining unrealistic designs.

The TOGW optimization did not change the planform area but increased the span from 58.7 to $62.3 \mathrm{~m}$ and increased the aspect ratio from 9.0 to 10.1. In contrast, the fuel-burn objective results in a significant increase in the wing span and wing area. In this case, the span has increased from 58.7 to $72.7 \mathrm{~m}$, the exposed planform area has increased by $16.2 \%$, and the new aspect ratio is 11.9 .

The most striking feature, however, is the addition of a raked wing tip similar to that of a Boeing 787. A smaller raked wing tip was also adopted in the higher-gross-weight versions of the Boeing 777. By including an additional sweep variable in the tip region, we facilitated the exploration of this design feature by the optimization, but the raked wing tip appears only when the objective is the TOGW. The

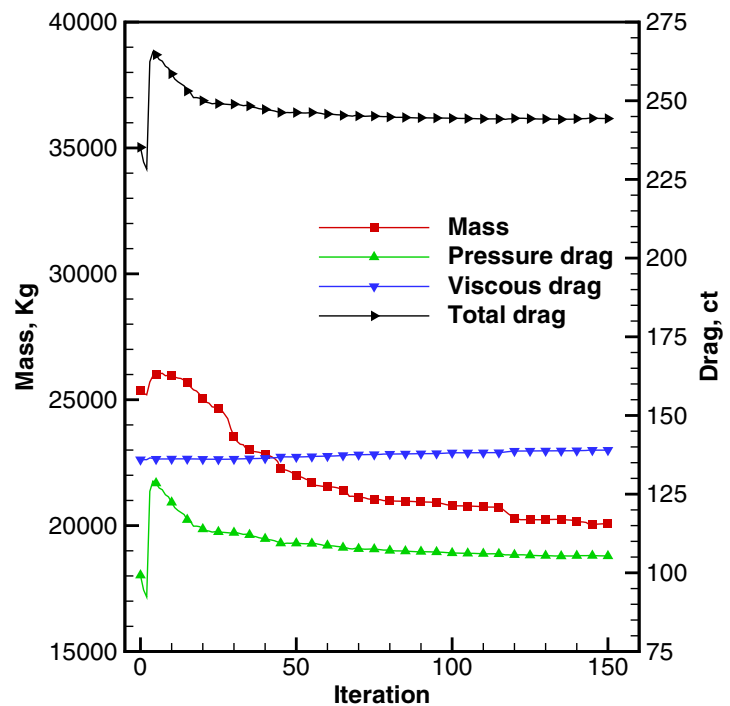

a) TOGW optimization benefit of the raked wing tip is that it passively alleviates the loads at the tip, as noted by McLean $[61,62]$ and further explained by Jansen et al. [63], who also obtained raked wing tips when performing aerostructural optimization with unconstrained spans. Because of the higher local sweep, the raked wing tip has a lower lift-curve slope, which means that the loads at the tip do not increase as much as those at the inboard section as the lift increases. In addition, the raked wing tip also has a larger moment arm in the streamwise direction, resulting in an additional aeroelastic downwash that further reduces the wingtip loads at the higher lift coefficients of the maneuver cases. Looking closely at the tip of the minimum fuel-burn design reveals that the leading edge is almost straight right at the tip, and there is a slight tapering of the chord near the tip.

The leading-edge sweep of the TOGW optimization remained essentially unchanged relative to the baseline except for the raked tip. The fuel-burn minimization results in a sweep that is more than $5 \mathrm{deg}$ lower than that of the original design. This reduction in sweep is required to enable the large span extension without incurring too large of a weight penalty. The wave-drag penalty for lowering the sweep angle is minimized through small shape modifications that can only be achieved with a large number of airfoil shape design variables. Additionally, the minimum fuel-burn optimum is thinner

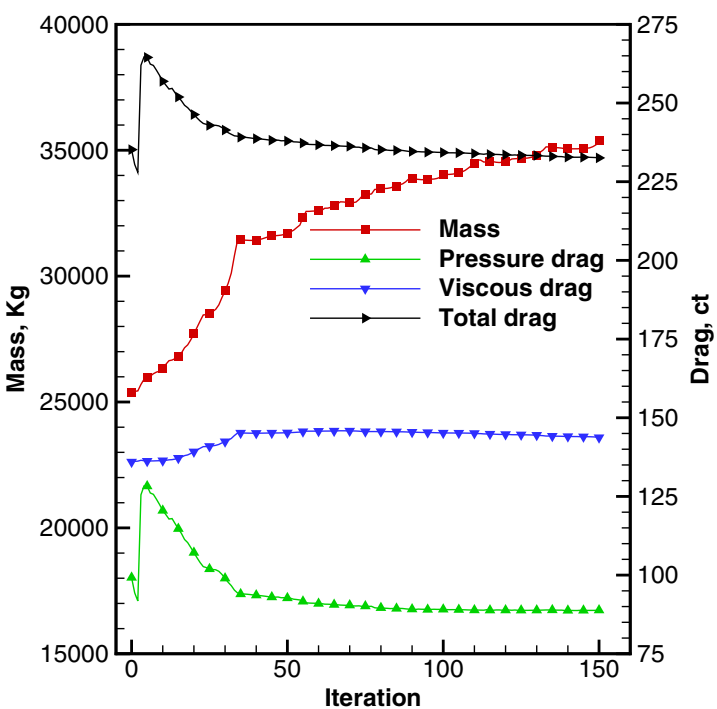

b) Fuel-burn optimization

Fig. 9 Weight and drag evolution during optimization (C1 condition). 


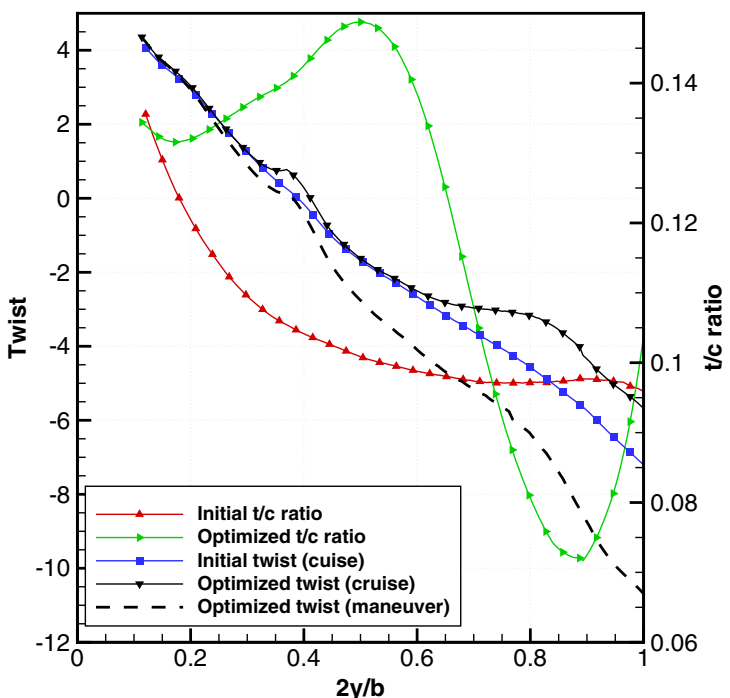

a) TOGW optimization

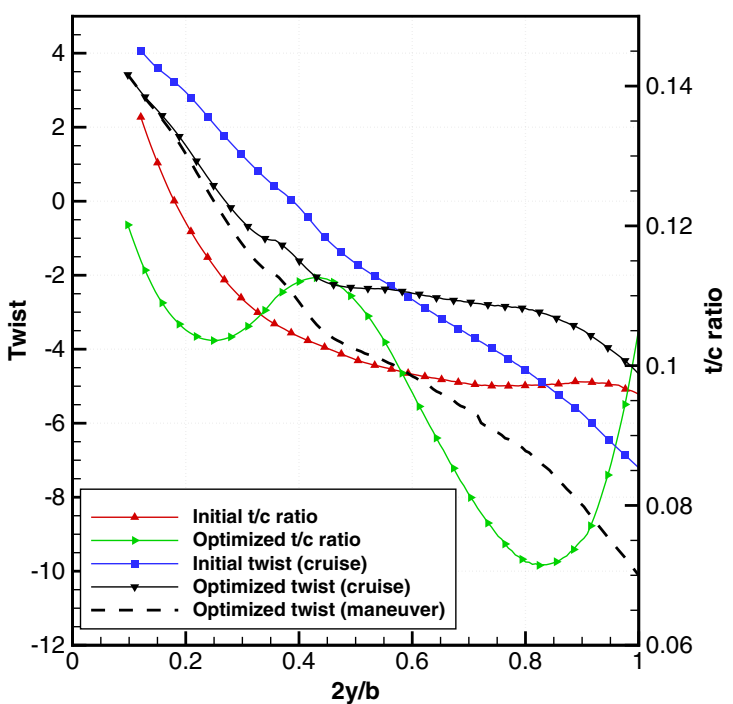

b) Fuel-burn optimization

Fig. 10 Twist and thickness-to-chord variation for the initial and optimized designs (C1 condition).

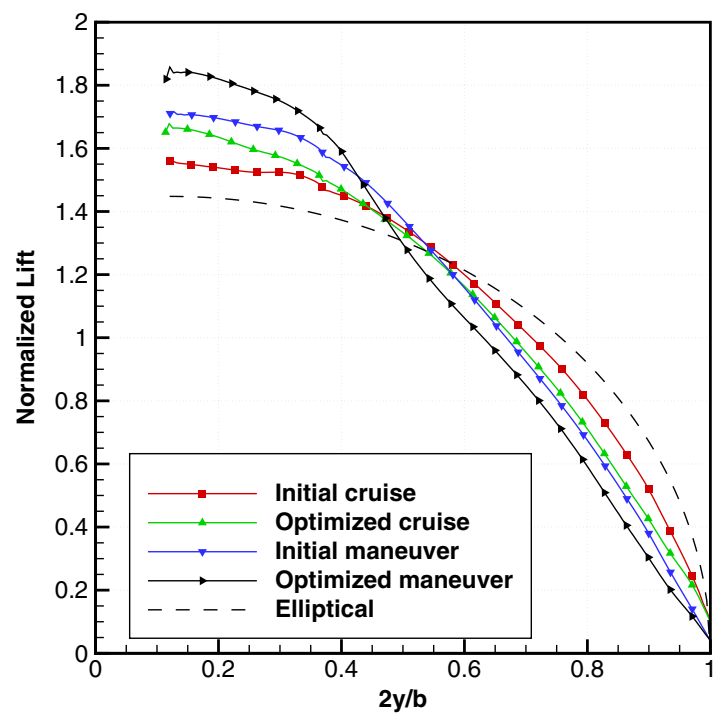

a) TOGW optimization

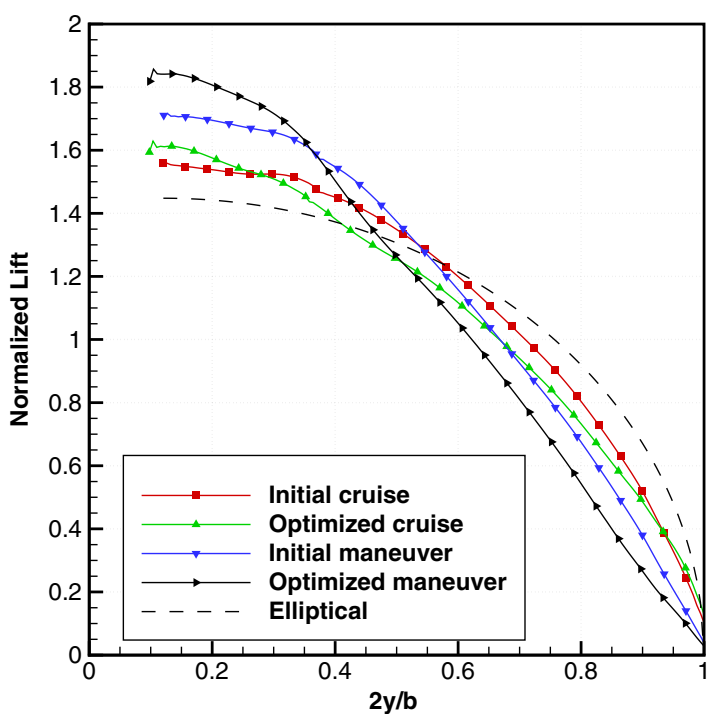

b) Fuel-burn optimization

Fig. 11 Spanwise lift distributions for the initial and optimized designs for $\mathrm{C} 1$ and $\mathrm{M} 1$ conditions.

than either the original or the TOGW optimum, which further limits the negative aspects of decreasing the sweep angle. These complex interactions can be predicted only with the high-fidelity aerostructural model used in this work.

We now evaluate the evolution of the inviscid pressure drag (which consists of induced drag and wave drag), the viscous drag, and the weight of the primary wing structure. The data are plotted in Fig. 9. Here, we can clearly see the vastly different tradeoffs made by each optimization to improve the chosen objective. The TOGW optimization slightly reduces the primary structural weight while simultaneously reducing the pressure drag. The viscous drag increases slightly, due primarily to significant overall increases in the $t / c$ ratio and reduction in sweep. The fuel-burn optimization, on the other hand, requires a significantly heavier structure to support the large span increase. In this case, the increase in the viscous drag is due primarily to the increase in the wing surface area because the $t / c$ ratios decreased slightly. Despite the increased structural weight and increased viscous drag, the total drag coefficient is lower for the fuel-burn objective.

An examination of the spanwise $t / c$ distribution and twist distributions, shown in Fig. 10, sheds additional light on the design tradeoffs in each optimization. The large difference between the

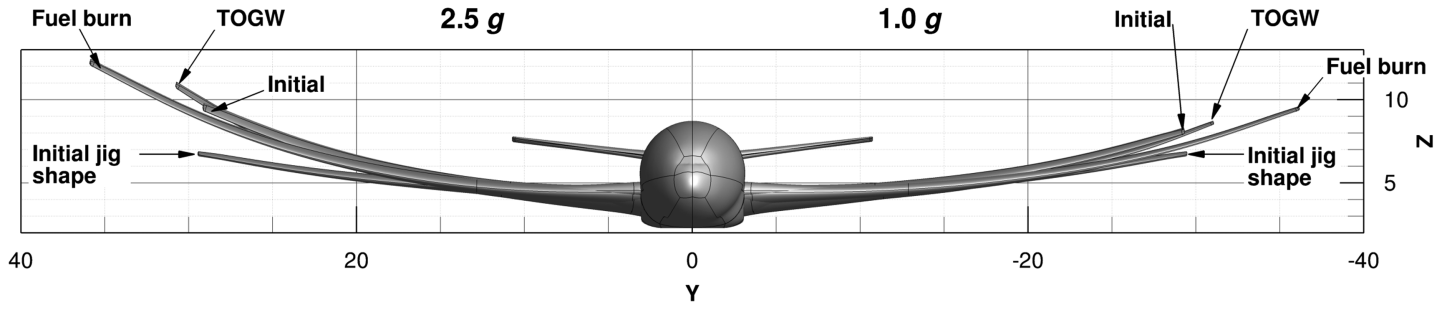

Fig. 12 Front view showing aerostructural deflections for M1 condition (left) and $\mathrm{C} 1$ condition (right). 
optimized $t / c$ ratios of the two optimized designs is immediately apparent. For the TOGW optimized design, the $t / c$ ratios have increased for most of the span, peaking at a maximum of $14.8 \%$ near the half-span position. For the fuel-burn optimization, an overall reduction in $t / c$ is observed with the exception of slight increases near and slightly outboard of the Yehudi break. There are two contributing factors to the increased primary structural weight for the fuel-burn optimized design: an increase in wing span, resulting in

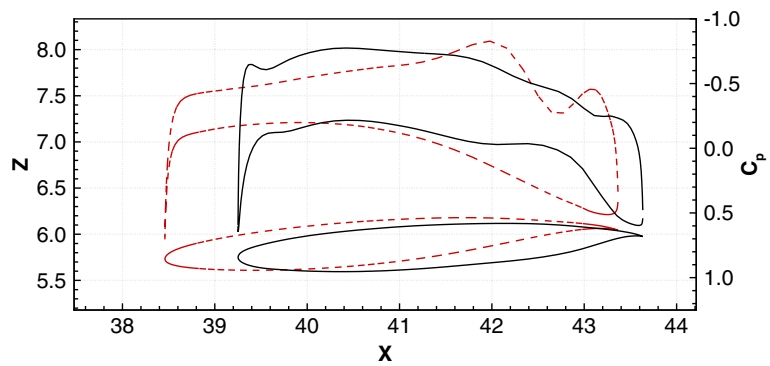

a) TOGW optimization, $\mathrm{C1}$

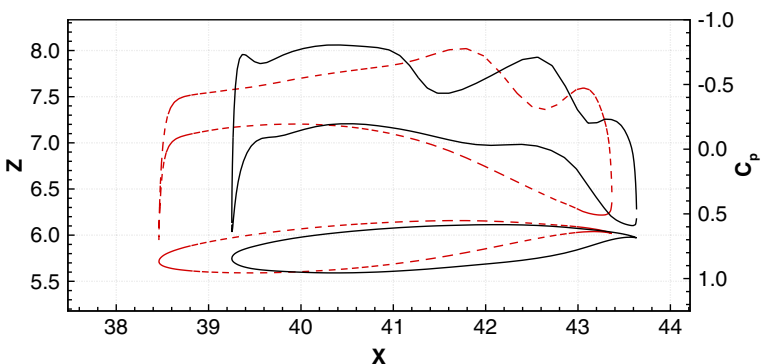

c) TOGW optimization, $\mathrm{C2}$

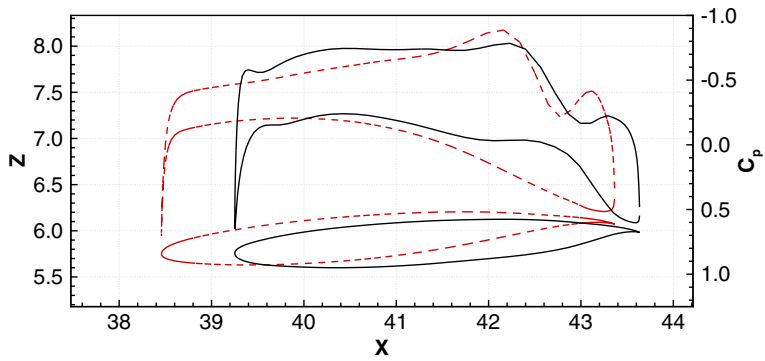

e) TOGW optimization, C3

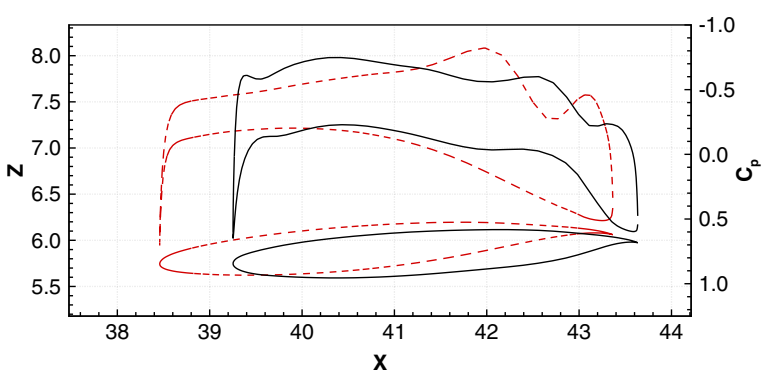

g) TOGW optimization, C4

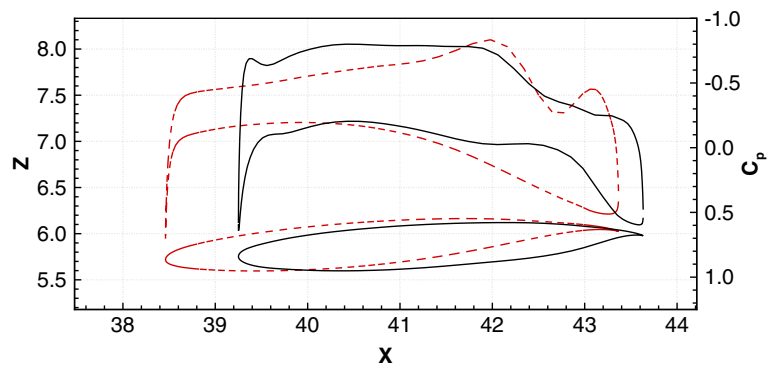

i) TOGW optimization, $\mathrm{C5}$

Fig. 13 Cross section and $C_{p}$ contours for each operating condition at $66 \%$ semispan: initial design (dashed lines) and optimized design (solid lines). higher bending moments, and a decrease in the $t / c$ distribution, resulting in a heavier structure. The twist distributions plotted in Fig. 10 are derived from the deformed flying shape of the wing. The optimized twist distributions generally follow the original distribution, with both designs reducing the amount of washout near the tip.

The spanwise lift distributions of the initial and optimized designs are shown in Fig. 11. The first observation is that, for all of the designs, the maneuver lift distributions have inboard-shifted load

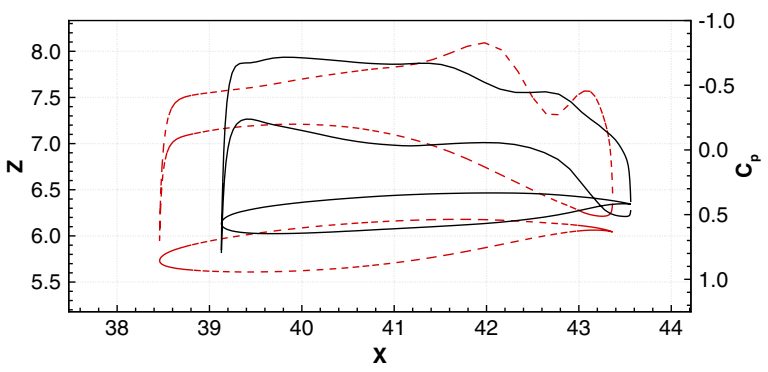

b) Fuel-burn optimization, C1

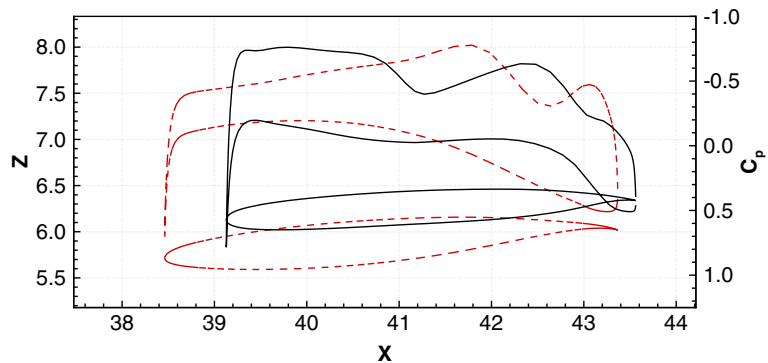

d) Fuel-burn optimization, C2

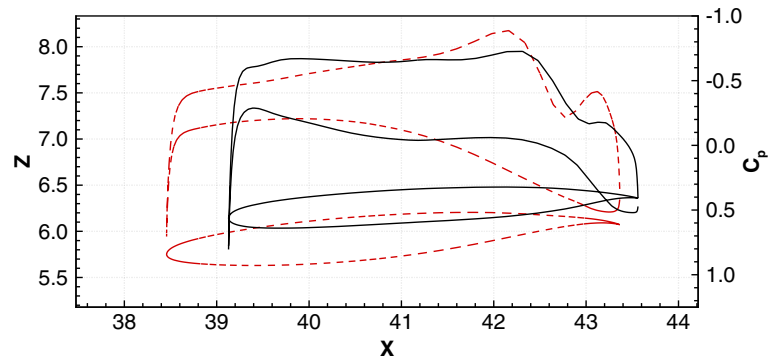

f) Fuel-burn optimization, C3

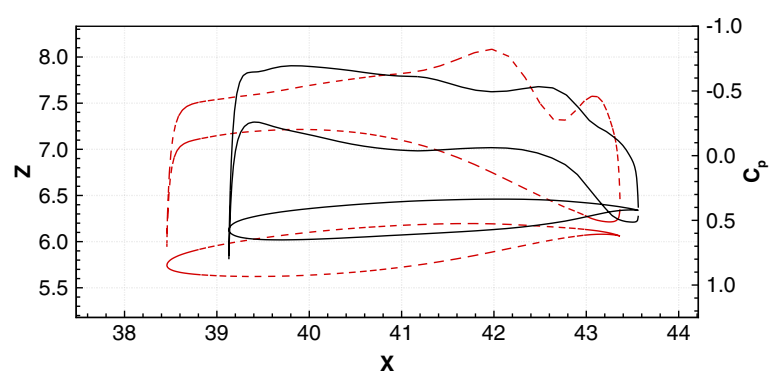

h) Fuel-burn optimization, C4

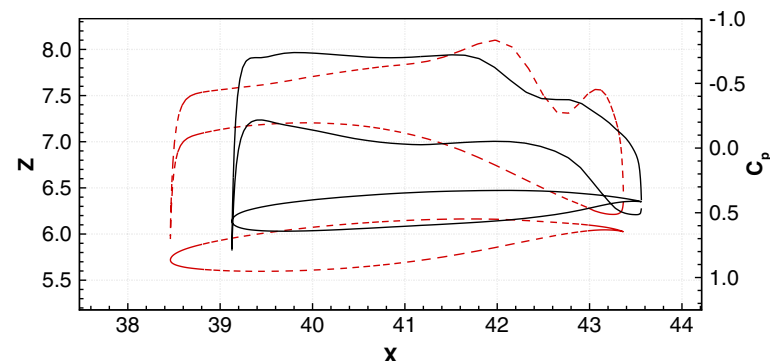

j) Fuel-burn optimization, C5 
distributions compared to the corresponding cruise lift distribution. This beneficial effect is caused by the aeroelastically induced washout near the tip, resulting in reduced loading, a form of passive static aeroelastic tailoring. The cruise lift distributions for each optimization show more triangular load distributions compared to the original or the elliptical reference. Although the difference is slight, the fuel-burn result has a lift distribution slightly closer to elliptical, resulting in a slight reduction in the induced drag. When we compare the lift distribution between the cruise and maneuver conditions, we see that the difference is more pronounced for the fuel-burn result. The TOGW optimum has a stiffer structure, and the raked wing tip, because of the lower lift-curve slope, is able to produce the beneficial inboard load shifting for the maneuver conditions. In the fuel-burn case, however, even though the wing is heavier, the increased span leads to larger deflections and a more consistent gradual twisting near the tip. A similar aeroelastic effect of reducing the tip load is achieved naturally without raking the tip. A comparison of the deflections of the initial and optimized designs as well as the jig shape is given in Fig. 12.

An examination of the chordwise $C_{p}$ distributions and airfoil shapes can explain the aerostructural tradeoffs that the optimizer

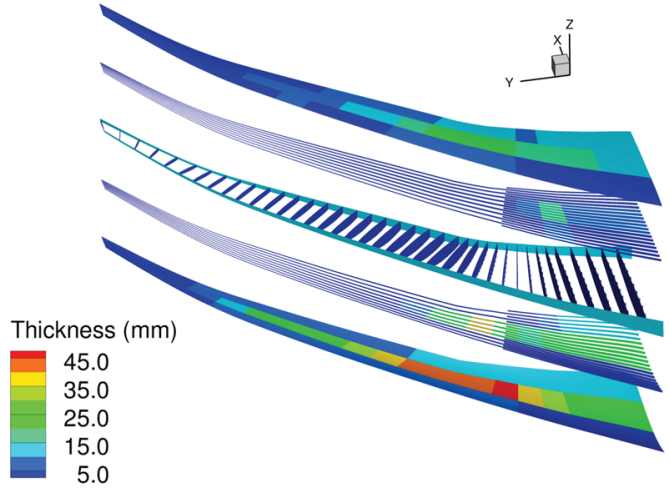

a) Thickness distribution for TOGW optimization

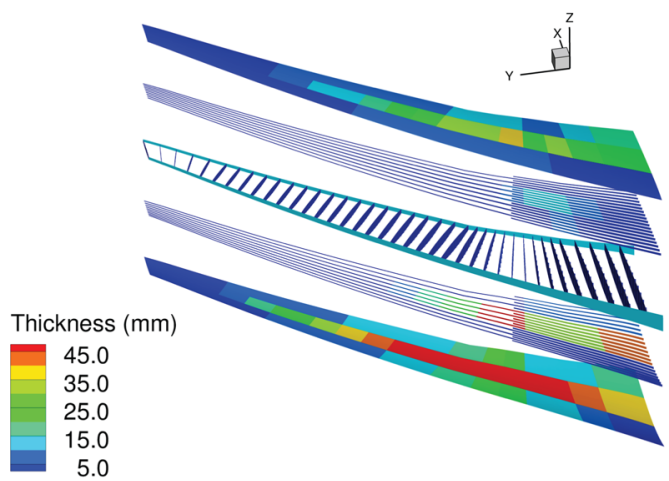

c) Thickness distribution for initial design

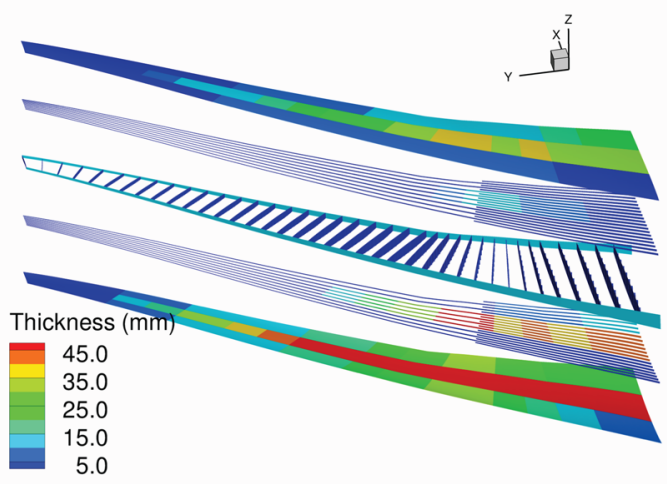

e) Thickness distribution for fuel-burn optimization made between the five cruise-design points and the two maneuver conditions. The cross-sectional data are extracted from the 3-D geometry using a cut plane orientated with the $x$ - $z$ plane. Figure 13 shows the airfoil shape and $C_{p}$ distribution for the initial and optimized designs for each operating condition at the $66 \%$ semispan location. Cruise conditions $\mathrm{C} 1, \mathrm{C} 4$, and $\mathrm{C} 5$ are all at the design cruise Mach number of 0.85 and all show a reduction in the shock strength on the wing upper surface, which lowers the wave drag. Condition $\mathrm{C} 2$, which corresponds to a lower Mach number of 0.84 , shows a distinct weak double shock structure on the upper-wing surface. The higher Mach number case of 0.86 (condition C3) shows a slightly reduced shock strength compared to the original.

The airfoils corresponding to the TOGW optimization are significantly thicker in the aft portion. This is primarily to accommodate a deeper, and thus more efficient, wing box structure. This helps to explain the weight reduction achieved for the TOGW optimization, despite the increase in wingspan. The thickening of the aft of the airfoil is also present in the fuel-burn optimization but to a lesser extent.

Because our aerodynamic model does not predict separation, it is likely that such airfoils would not perform well in the real world.

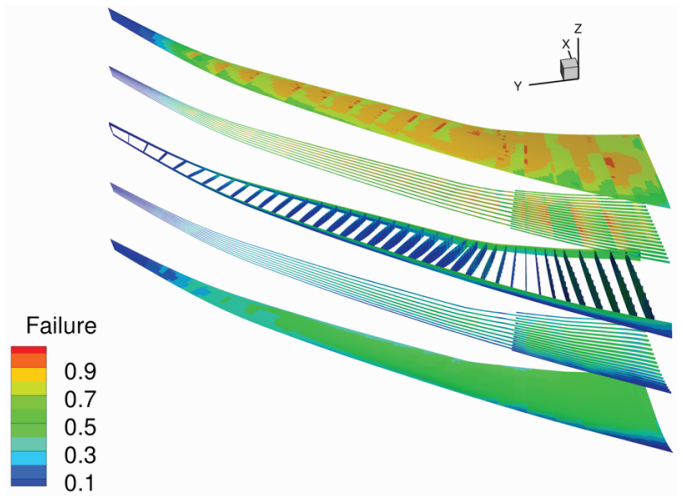

b) Failure distribution for TOGW optimization, condition M1

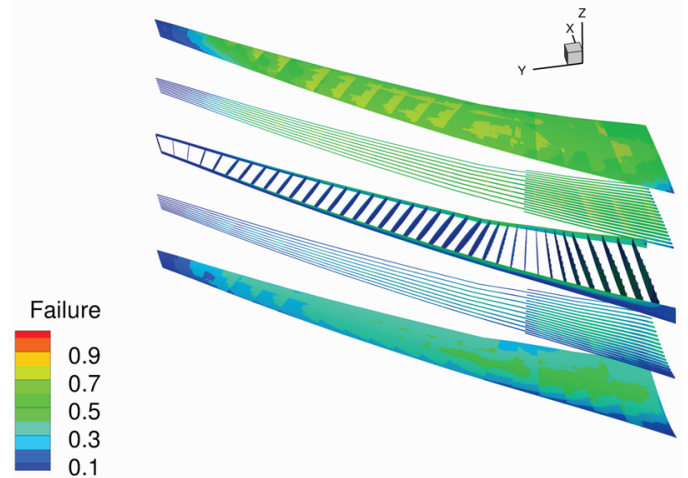

d) Failure distribution for initial design, condition M1

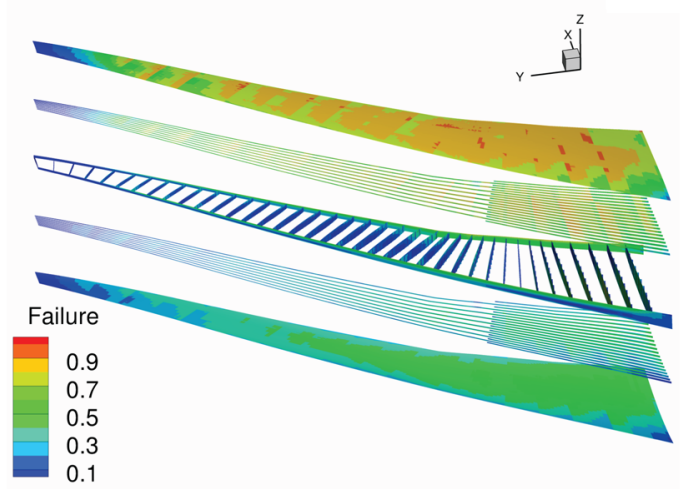

f) Failure distribution for fuel-burn optimization, condition M1

Fig. 14 Initial and optimized thickness (left column) and failure (right column) distributions over the wing box. 
Thus, we have since implemented the solution of the RANS equations [21,22]. The RANS equations are expected to yield a more accurate prediction of the wave drag (because the shock location and strength are strongly dependent on viscous effects) and the viscous drag (both the pressure and skin-friction components). The improved model would also correctly penalize designs with significant flow separation, particularly in cases where the separation is induced by shocks.

Figure 14 shows the structural design sizing resulting from the two aerostructural optimizations. The structural failure parameter is the ratio of the von Mises stress to the yield stress of the material. Recall that the initial design was first optimized with fixed aerostructural loads, and that this weight minimization resulted in a failure distribution that meets the KS failure constraints, which is similar to Figs. $14 \mathrm{~b}$ and $14 \mathrm{f}$. However, when this design is used in the multidisciplinary optimization, we observe lower stress values. This highlights the need for MDO; even though we performed a structural optimization, we would have to continue the procedure of generating loads from the previous design and re-optimizing to generate a design that meets the multidisciplinary constraint.

Each optimization resulted in similar failure distributions. The plots in Fig. 14 show the failure load for the symmetric $2.5 \mathrm{~g}$ pull-up maneuver. The upper skin and stringers are critical for this load case, but the lower surface is not; the lower skin and stringers are dimensioned by the $1.3 \mathrm{~g}$ gust load. The overall distribution of material for the initial design and the two optimized designs is similar. The largest skin thickness occurs through the midsection of the spar box, where the thickness is greatest. The TOGW optimum shows a locally increased physical wing thickness near the Yehudi break of the wing, even with the large increase in $t / c$ that we described earlier. To support loading from the span extension, the fuel-burn results show significantly increased thicknesses over the majority of the lower skin and stringers.

Finally, Table $\underline{6}$ lists the key results from the optimizations. We achieve drag reduction for all operating conditions for both optimizations, with the higher Mach number point $\mathrm{C} 2$ and the higher lift condition $\mathrm{C} 5$ resulting in the largest improvements.

For the fuel-burn optimization, the pressure drag was reduced by $30 \%$. As for the TOGW optimization, there is a reduction in the wave drag, but there is a much greater reduction in the induced drag due to the higher aspect ratio. In fact, the TOGW for the fuel-burn optimization remained essentially unchanged; the increase in the structural weight is offset by the reduced fuel load.

The weight breakdown of the primary structure, shown in Table $\underline{6}$, shows where the weight is saved in the TOGW optimization. The largest weight reduction is from the top and bottom skins as well as the lower stringers. For the lower skin and stringers, the gust load is critical. From Fig. 11, even the $1 g$ cruise conditions have a more heavily loaded inboard lift distribution than that of the initial design. Because the $1.3 \mathrm{~g}$ lift distribution is similar to the cruise condition, we can conclude that the slight induced drag penalty from a more linear lift distribution is offset by the weight reduction in the lower skin and stringers. For the fuel-burn optimization, we see weight increases across almost all of the components, with the largest increases in the skins and rear spar. Given the reduced $t / c$ and increased span, these increases are expected.

The structural analysis in this work considered only a linear structural response. The large deflections observed for the $2.5 g$ maneuver condition call this assumption into question. TACS is capable of analyzing a geometrically nonlinear structural response, and this could be incorporated in the future. Because the rib and stringer pitches increase with increases in span and chord,

Table 6 Optimization results summary

\begin{tabular}{|c|c|c|c|c|c|}
\hline \multirow[t]{2}{*}{ Quantity } & \multirow{2}{*}{$\begin{array}{l}\text { Initial } \\
\text { Value }\end{array}$} & \multicolumn{2}{|c|}{ TOGW optimization } & \multicolumn{2}{|c|}{ Fuel-burn optimization } \\
\hline & & Value & Percent change, $\%$ & Value & Percent change, $\%$ \\
\hline Pressure drag $\mathrm{C} 1$, count & 134.0 & 105.3 & -21.4 & 88.9 & -33.7 \\
\hline Pressure drag $\mathrm{C} 2$, count & 133.1 & 112.6 & -15.4 & 93.2 & -30.0 \\
\hline Pressure drag $\mathrm{C} 3$, count & 143.4 & 111.5 & -22.2 & 93.8 & -34.6 \\
\hline Pressure drag $\mathrm{C} 4$, count & 122.7 & 99.4 & -19.0 & 83.7 & -31.8 \\
\hline Pressure drag C5, count & 147.7 & 114.3 & -22.2 & 95.9 & -35.1 \\
\hline Average pressure drag, count & 136.0 & 108.6 & -20.2 & 91.1 & -33.1 \\
\hline Viscous drag, count & 136.0 & 139.0 & 2.2 & 143.7 & 5.7 \\
\hline Mass bottom skin, kg & 12,653 & 9,192 & -27.4 & 18,583 & 46.9 \\
\hline Mass bottom stringers, $\mathrm{kg}$ & 2,055 & 1,687 & -17.9 & 2,539 & 23.6 \\
\hline Mass top skin, $\mathrm{kg}$ & 7,519 & 5,424 & -27.9 & 10,545 & 40.3 \\
\hline Mass top stringers, $\mathrm{kg}$ & 557 & 691 & 24.0 & 634 & 13.8 \\
\hline Mass ribs, $\mathrm{kg}$ & 581 & 578 & -0.5 & 578 & -0.5 \\
\hline Mass rib stiffeners, $\mathrm{kg}$ & 45 & 56 & 24.5 & 51 & 14.4 \\
\hline Mass front spar, $\mathrm{kg}$ & 1,235 & 1,307 & 5.8 & 1,387 & 12.4 \\
\hline Mass rear spar, $\mathrm{kg}$ & 723 & 1,145 & 58.3 & 1,049 & 45.1 \\
\hline Total wing mass, $\mathrm{kg}$ & 25,368 & 20,078 & -20.8 & 35,368 & 39.4 \\
\hline Final cruise weight, kg & 187,071 & 181,779 & -2.8 & 198,367 & 6.0 \\
\hline Initial cruise $\mathrm{C} 1$ mass, $\mathrm{kg}$ & 289,203 & 276,875 & -4.3 & 289,148 & 0.0 \\
\hline Initial cruise $\mathrm{C} 2$ mass, $\mathrm{kg}$ & 287,321 & 278,618 & -3.0 & 289,886 & 0.9 \\
\hline Initial cruise $\mathrm{C} 3$ mass, $\mathrm{kg}$ & 295,213 & 280,218 & -5.1 & 291,991 & -1.1 \\
\hline Initial cruise $\mathrm{C} 4$ mass, $\mathrm{kg}$ & 290,405 & 278,340 & -4.2 & 290,729 & 0.1 \\
\hline Initial cruise $\mathrm{C} 5$ mass, $\mathrm{kg}$ & 288,858 & 276,411 & -4.3 & 288,201 & -0.2 \\
\hline Average cruise mass & 290,200 & 278,098 & -4.2 & 289,991 & -0.1 \\
\hline C1 Fuel burn, $\mathrm{kg}$ & 102,132 & 95,097 & -6.9 & 90,782 & -11.1 \\
\hline C2 Fuel burn, kg & 100,250 & 96,839 & -3.4 & 91,520 & -8.7 \\
\hline C3 Fuel burn, kg & 108,141 & 98,440 & -9.0 & 93,625 & -13.4 \\
\hline C4 Fuel burn, kg & 103,334 & 96,561 & -6.6 & 92,362 & -10.6 \\
\hline C5 Fuel burn, kg & 101,789 & 96,320 & -7.0 & 89,834 & -11.7 \\
\hline Average fuel burn, kg & 103,129 & 96,320 & -6.6 & 91,625 & -11.2 \\
\hline Span, $\mathrm{m}$ & 58.6 & 62.1 & 6.0 & 72.3 & 23.4 \\
\hline Aspect ratio & 9.0 & 10.1 & 12.2 & 11.9 & 32.2 \\
\hline Leading-edge sweep, deg & 37.4 & 37.6 & 0.1 & 32.0 & -14.4 \\
\hline Reference wing area, $\mathrm{m}^{2}$ & 383.7 & 383.7 & 0.0 & 437.9 & 14.1 \\
\hline Exposed wing area, $\mathrm{m}^{2}$ & 335.0 & 335.0 & 0.0 & 389.2 & 16.2 \\
\hline Wing volume, $\mathrm{m}^{3}$ & 46.0 & 53.0 & 15.2 & 48.7 & 5.9 \\
\hline
\end{tabular}


respectively, we would like to include buckling constraints to more accurately capture the weight increase due to these changes. Because composite structures are playing an increasingly important role in commercial aircraft, we would also like to consider composite wing box structures, as done in previous work with lower-fidelity aerodynamics by Kennedy and Martins [41].

Finally, as we begin to examine more operating conditions, it becomes increasingly important to decide how to combine the performance parameters from each analysis into a composite objective function that is realistic. A weighted average can be used to assign more importance to certain operating conditions, but it is not straightforward to select these weights and to measure their effect on the optimum design. We are currently investigating the use of surrogate models to approximate the aerostructural performance in the cruise regime to allow more complex mission analysis and incorporate actual aircraft usage patterns into the objective formulation [64].

\section{Conclusions}

In this paper, an approach for obtaining optimal static aeroelastic tailoring of aircraft wings was presented. Aerostructural optimizations of the NASA CRM geometry were performed with a structure created to be representative of a modern airliner wing. Multipoint optimizations with five cruise conditions and two maneuver conditions were performed with a 2 million cell CFD mesh and 300,000-degree-of-freedom structural mesh. The optimization problems were solved with respect to 476 design variables subject to 57 geometric, trim, and stress constraints. The solution of these problems required $36 \mathrm{~h}$ of wall time using 435 processors.

The solution of these high-fidelity, high-dimensional design problems was enabled by a scalable parallel aerostructural solver and the combination of an efficient method for computing coupled derivatives together with a state-of-the-art gradient-based optimizer. TOGW and fuel burn were minimized in separate design optimization problems, providing insights into the aerostructural tradeoffs of transonic wing design.

A sensitivity analysis of each objective function showed that the fuel-burn objective should result in an optimum with lower drag than the TOGW objective. The optimized results show that this is indeed the case: the fuel-burn optimization reduced the cruise-segment fuel consumption by $11.2 \%$, while the TOGW optimization reduced the fuel burn by $6.6 \%$. Conversely, the TOGW minimization resulted in a $4.2 \%$ reduction in the TOGW, while the TOGW for the fuel-burn optimization remained practically unchanged.

The minimum TOGW configuration exhibited a raked wing tip similar to that seen in the Boeing 787, which shifts the spanwise lift distribution inboard at the maneuver conditions. This is a form of passive load alleviation that can only be found by simultaneous consideration of the aerodynamics and structures. The minimum fuel burn resulted in a wing with a span 18\% larger than the TOGW one. This increase in span came at the cost of $76 \%$ higher wing structure mass but ultimately reduced the fuel burn relative to the TOGW case by $4.9 \%$.

The optimization results demonstrate that the proposed approach performs tradeoffs between aerodynamic and structural performance in an effective way, taking into account the chosen objective. We are now capable of performing the aerostructural design optimization of full configurations with respect to hundreds of aerodynamic shape and structural sizing design variables, subject to real-world constraints. This is a promising approach for designing the wings of future aircraft, which are expected to exhibit large-span wings that are more flexible. This trend in larger-span wings is likely to continue as the price of fuel increases (shifting the tradeoff to higher-mass, lowerinduced-drag wings) and as better materials and structural designs emerge (enabling larger span for a given structural mass). This shift in the aerostructural tradeoffs will change the design space, and the approach proposed in this paper provides an effective tool to explore this new design space, while taking full advantage of static aeroelastic tailoring.

\section{Acknowledgments}

The authors are grateful for the funding provided by the Natural Sciences and Engineering Research Council. The computations were performed on the General Purpose Cluster (GPC) supercomputer at the SciNet High Performance Computing Consortium. SciNet is funded by the Canada Foundation for Innovation under the auspices of Compute Canada; the Government of Ontario; the Ontario Research Fund - Research Excellence; and the University of Toronto.

\section{References}

[1] Ashley, H., "On Making Things the Best-Aeronautical Uses of Optimization," Journal of Aircraft, Vol. 19, No. 1, Jan. 1982, pp. 5-28. doi: $10.2514 / 3.57350$

[2] Sobieszczanski-Sobieski, J., and Haftka, R. T., "Multidisciplinary Aerospace Design Optimization: Survey of Recent Developments," Structural Optimization, Vol. 14, No. 1, 1997, pp. 1-23. doi:10.1007/BF01197554

[3] Martins, J. R. R. A., and Lambe, A. B., "Multidisciplinary Design Optimization: A Survey of Architectures," AIAA Journal, Vol. 51, No. 9 , 2013, pp. 2049-2075. doi:10.2514/1.J051895

[4] Pai, S. I., and Sears, W. R., "Some Aeroelastic Properties of Swept Wings," Journal of the Aeronautical Sciences, Vol. 16, No. 2, 1949, pp. 105-115.

[5] Brown, H., "A Method for the Determination of the Spanwise Load Distribution of a Flexible Swept Wing at Subsonic Speeds," Defense Technical Information Center, TN-2222, Fort Belvoir, VA, 1951.

[6] Diederich, F. W., "Calculation of the Aerodynamic Loading of Flexible Wings of Arbitrary Planform and Stiffness," NACA TN-1876, Langley, VA, 1949.

[7] Livne, E., "Future of Airplane Aeroelasticity," Journal of Aircraft, Vol. 40, No. 6, 2003, pp. 1066-1092. doi: $10.2514 / 2.7218$

[8] Haftka, R. T., "Optimization of Flexible Wing Structures Subject to Strength and Induced Drag Constraints," AIAA Journal, Vol. 14, No. 8, 1977, pp. 1106-1977.

[9] Grossman, B., Strauch, G., Eppard, W. H., Gürdal, Z., and Haftka, R. T., "Integrated Aerodynamic/Structural Design of a Sailplane Wing," Journal of Aircraft, Vol. 25, No. 9, 1988, pp. 855-860. doi: $10.2514 / 3.45670$

[10] Grossman, B., Haftka, R. T., Kao, P.-J., Polen, D. M., and Rais-Rohani, M., "Integrated Aerodynamic-Structural Design of a Transport Wing," Journal of Aircraft, Vol. 27, No. 12, 1990, pp. 1050-1056. doi: $10.2514 / 3.45980$

[11] Chittick, I. R., and Martins, J. R. R. A., "An Asymmetric Suboptimization Approach to Aerostructural Optimization," Optimization and Engineering, Vol. 10, No. 1, 2009, pp. 133-152. doi:10.1007/s11081-008-9046-2

[12] Schmit, L. A., "Structural Design by Systematic Synthesis," Proceedings of the 2nd Conference on Electronic Computation, American Society of Civil Engineers, Reston, VA, 1960, pp. 105-132.

[13] Venkayya, V. B., "Structural Optimization: A Review and Some Recommendations," International Journal for Numerical Methods in Engineering, Vol. 13, No. 2, 1978, pp. 203-228. doi:10.1002/(ISSN)1097-0207

[14] Venkatamaran, S., and Haftka, R. T., "Structural Optimization Complexity: What Has Moore's Law Done for Us?," Structural and Multidisciplinary Optimization, Vol. 28, No. 6, 2004, pp. 375-387. doi: $10.1007 / \mathrm{s} 00158-004-0415-\mathrm{y}$

[15] Jameson, A., "Aerodynamic Design via Control Theory," Journal of Scientific Computing, Vol. 3, No. 3, 1988, pp. 233-260. doi:10.1007/BF01061285

[16] Cliff, S. E., Reuther, J. J., Saunders, D. A., and Hicks, R. M., "SinglePoint and Multipoint Aerodynamic Shape Optimization of High-Speed Civil Transport," Journal of Aircraft, Vol. 38, No. 6, 2001, pp. 9971005 .

doi: $10.2514 / 2.2886$

[17] Hicken, J. E., and Zingg, D. W., "Aerodynamic Optimization Algorithm with Integrated Geometry Parameterization and Mesh Movement," AIAA Journal, Vol. 48, No. 2, Feb. 2009, pp. 400-413. doi: $10.2514 / 1.44033$

[18] Mader, C. A., and Martins, J. R. R. A., "Stability-Constrained Aerodynamic Shape Optimization of Flying Wings," Journal of Aircraft, Vol. 50, No. 5, 2013, pp. 1431-1449. doi:10.2514/1.C031956 
[19] Leoviriyakit, K., and Jameson, A., "Multipoint Wing Planform Optimization via Control Theory," 43rd AIAA Aerospace Sciences Meeting and Exhibit, AIAA Paper 2005-0450, Feb. 2005.

[20] Dwight, R. P., and Brezillon, J., "Efficient and Robust Algorithms for Solution of the Adjoint Compressible Navier-Stokes Equations with Applications," International Journal for Numerical Methods in Fluids, Vol. 60, No. 4, 2009, pp. 355-471.

doi:10.1002/fld.v60:4

[21] Lyu, Z., Kenway, G. K., Paige, C., and Martins, J. R. R. A., "Automatic Differentiation Adjoint of the Reynolds-Averaged Navier-Stokes Equations with a Turbulence Model," 21st AIAA Computational Fluid Dynamics Conference, San Diego, CA, July 2013.

[22] Lyu, Z., and Martins, J. R. R. A., "RANS-Based Aerodynamic Shape Optimization of a Blended-Wing-Body Aircraft," 21st AIAA Computational Fluid Dynamics Conference, AIAA Paper 2013-2586, July 2013.

[23] Haug, E. J., and Feng, T.-T., "Optimal Design of Dynamically Loaded Continuous Structures," International Journal for Numerical Methods in Engineering, Vol. 12, No. 2, 1978, pp. 299-317. doi:10.1002/(ISSN)1097-0207

[24] Pironneau, O., "On Optimum Design in Fluid Mechanics," Journal of Fluid Mechanics, Vol. 64, No. 01, 1974, pp. 97-110. doi:10.1017/S0022112074002023

[25] Peter, J. E. V., and Dwight, R. P., "Numerical Sensitivity Analysis for Aerodynamic Optimization: A Survey of Approaches," Computers and Fluids, Vol. 39, No. 3, 2010, pp. 373-391. doi:10.1016/j.compfluid.2009.09.013

[26] Brown, S. A., "Displacement Extrapolation for CFD+CSM Aeroelastic Analysis," AIAA Paper 1997-1090, 1997.

[27] Maman, N., and Farhat, C., "Matching Fluid and Structure Meshes for Aeroelastic Computations: A Parallel Approach," Computers and Structures, Vol. 54, No. 4, 1995, pp. 779-785. doi:10.1016/0045-7949(94)00359-B

[28] Farhat, C., Lesoinnea, M., and LeTallec, P., "Load and Motion Transfer Algorithms for Fluid/Structure Interaction Problems with NonMatching Discrete Interfaces: Momentum and Energy Conservation, Optimal Discretization and Application to Aeroelasticity," Computer Methods in Applied Mechanics and Engineering, Vol. 157, Nos. 1-2, 1996, pp. 95-115.

[29] Kamakoti, R., and Shyy, W., "Fluid-Structure Interaction for Aeroelastic Applications," Progress in Aerospace Sciences, Vol. 40, No. 8, 2004, pp. 535-558.

doi:10.1016/j.paerosci.2005.01.001

[30] Felippa, C. A., Park, K., and Farhat, C., "Partitioned Analysis of Coupled Mechanical Systems," Computer Methods in Applied Mechanics and Engineering, Vol. 190, Nos. 24-25, 2001, pp. 32473270 .

doi:10.1016/S0045-7825(00)00391-1

[31] Kim, J. Y., Aluru, N. R., and Tortorelli, D. A., "Improved Multi-Level Newton Solvers for Fully Coupled Multi-Physics Problems," International Journal for Numerical Methods in Engineering, Vol. 58, No. 3, 2003, pp. 463-480. doi:10.1002/nme.751

[32] Martins, J. R. R. A., Alonso, J. J., and Reuther, J. J., "Aero-Structural Wing Design Optimization Using High-Fidelity Sensitivity Analysis," Proceedings of the CEAS Conference on Multidisciplinary Aircraft Design and Optimization, edited by Hölinger, H., Cologne, Germany, June 2001, pp. 211-226.

[33] Martins, J. R. R. A., Alonso, J. J., and Reuther, J. J., "A Coupled-Adjoint Sensitivity Analysis Method for High-Fidelity Aero-Structural Design," Optimization and Engineering, Vol. 6, No. 1, 2005, pp. 33-62. doi:10.1023/B:OPTE.0000048536.47956.62

[34] Martins, J. R. R. A., Alonso, J. J., and Reuther, J. J., "High-Fidelity Aerostructural Design Optimization of a Supersonic Business Jet," Journal of Aircraft, Vol. 41, No. 3, 2004, pp. 523-530. doi: $10.2514 / 1.11478$

[35] Kenway, G. K. W., Kennedy, G. J., and Martins, J. R. R. A., “A Scalable Parallel Approach for High-Fidelity Steady-State Aeroelastic Analysis and Derivative Computations," AIAA Journal (to be published).

[36] Martins, J. R. R. A., and Hwang, J. T., "Review and Unification of Methods for Computing Derivatives of Multidisciplinary Systems," AIAA Journal, Vol. 51, No. 11, 2013, pp. 2582-2599. doi:10.2514/1.J052184

[37] Maute, K., Nikbay, M., and Farhat, C., "Sensitivity Analysis and Design Optimization of Three-Dimensional Non-Linear Aeroelastic Systems by the Adjoint Method," International Journal for Numerical Methods in Engineering, Vol. 56, No. 6, 2003, pp. 911-933. doi:10.1002/(ISSN)1097-0207

[38] Barcelos, M., Bavestrello, H., and Maute, K., "A Schur-NewtonKrylov Solver for Steady-State Aeroelastic Analysis and Design
Sensitivity Analysis," Computer Methods in Applied Mechanics and Engineering, Vol. 195, Nos. 17-18, 2006, pp. 2050-2069. doi:10.1016/j.cma.2004.09.013

[39] Brezellion, J., Ronzheimer, A., Haar, D., Abu-Zurayk, M., Lummer, K., Krugër, W., and Nattere, F. J., "Development and Application of MultiDisciplinary Optimization Capabilities Based on High-Fidelity Methods," AIAA Paper 2012-1757, April 2012.

[40] Ghazlane, I., Carrier, G., Dumont, A., and Désidéri, J.-A., "Aerostructural Adjoint Method for Flexible Wing Optimization," AIAA Paper 2012-1924, April 2012.

[41] Kennedy, G. J., and Martins, J. R. R. A., "A Comparison of Metallic and Composite Aircraft Wings Using Aerostructural Design Optimization," AIAA Paper 2012-5475, Sept. 2012.

[42] Kennedy, G. J., and Martins, J. R. R. A., "A Laminate Parametrization Technique for Discrete Ply Angle Problems with Manufacturing Constraints," Structural and Multidisciplinary Optimization, Vol. 48, No. 2, Aug. 2013, 379-393. doi:10.1007/s00158-013-0906-9

[43] Piperni, P., Abdo, M., Kafyeke, F., and Isikveren, A. T., "Preliminary Aerostructural Optimization of a Large Business Jet," Journal of Aircraft, Vol. 44, No. 5, 2007, pp. 1422-1438. doi:10.2514/1.26989

[44] DeBlois, A., and Abdo, M., "Multi-Fidelity Multidisciplinary Design Optimization of Metallic and Composite Regional and Business Jets," AIAA Paper 2010-9191, 2011.

[45] van der Weide, E., Kalitzin, G., Schluter, J., and Alonso, J. J., "Unsteady Turbomachinery Computations Using Massively Parallel Platforms," AIAA Paper 2006-0421, 2006.

[46] Kennedy, G. J., and Martins, J. R. R. A., "Parallel Solution Methods for Aerostructural Analysis and Design Optimization," AIAA Paper 20109308, Sept. 2010.

[47] Hopkins, E. J., "Charts for Predicting Turbulent Skin Friction form the Van Driest Method (II)," NASA TN-D-6945, 1972

[48] Mason, W., "Program FRICTION Manual," Virginia Polytechnic Inst. and State Univ., Blacksburg, VA, http://www.dept.aoe.vt.edu/ mason/ Mason_f/MRsoft.html [retrieved Nov. 2010].

[49] Torenbeek, E., Synthesis of Subsonic Airplane Design, 6th ed., Delft Univ. Press, Delft, The Netherlands, 1990, p. 499.

[50] Wrenn, G., "An Indirect Method for Numerical Optimization Using the Kreisselmeier-Steinhauser Function,” NASA TR-4220, 1989.

[51] Vassberg, J. C., DeHaan, M. A., Rivers, S. M., and Wahls, R. A., "Development of a Common Research Model for Applied CFD Validation Studies," AIAA Paper 2008-6919, Aug. 2008.

[52] “777-200/300 Airplane Characteristics for Airport Planning," Boeing Commercial Airplanes, Rept. D6-58329, Renton, WA, July 1998.

[53] Nemec, M., Zingg, D. W., and Pulliam, T. H., "Multipoint and MultiObjective Aerodynamic Shape Optimization," AIAA Journal, Vol. 42, No. 6, 2004, pp. 1057-1065. doi: $10.2514 / 1.10415$

[54] Hürlimann, F., "Mass Estimation of Transport Aircraft Wing," Ph.D. Thesis, Swiss Federal Inst. of Technology Zurich, Zurich, 2010.

[55] Kenway, G. K. W., Kennedy, G. J., and Martins, J. R. R. A., "A CADFree Approach to High-Fidelity Aerostructural Optimization," AIAA Paper 2010-9231, Sept. 2010.

[56] Dvorkin, E. N., and Bathe, K.-J., "A Continuum Mechanics Based FourNode Shell Element for General Nonlinear Analysis," Engineering Computations, Vol. 1, No. 1, 1984, pp. 77-88. doi:10.1108/eb023562

[57] Poon, N. M. K., and Martins, J. R. R. A., "An Adaptive Approach to Constraint Aggregation Using Adjoint Sensitivity Analysis," Structures and Multidisciplinary Optimization, Vol. 30, No. 1, 2007, pp. 61-73. doi:10.1007/s00158-006-0061-7

[58] Gill, P. E., Murray, W., and Saunders, M. A., "SNOPT: An SQP Algorithm for Large-Scale Constrained Optimization," SIAM Review, Vol. 47, No. 1, 2005, pp. 99-131. doi: $10.1137 /$ S0036144504446096

[59] Perez, R. E., Jansen, P. W., and Martins, J. R. R. A., "pyOpt: A PythonBased Object-Oriented Framework for Nonlinear Constrained Optimization," Structural and Multidisciplinary Optimization, Vol. 45, No. 1, Jan. 2012, pp. 101-118. doi:10.1007/s00158-011-0666-3

[60] Loken, C., Gruner, D., Groer, L., Peltier, R., Bunn, N., Craig, M., Henriques, T., Dempsey, J., Yu, C.-H., Chen, J., Dursi, L. J., Chong, J., Northrup, S., Pinto, J., Knecht, N., and Zon, R. V., "SciNet: Lessons Learned from Building a Power-Efficient Top-20 System and Data Centre," Journal of Physics: Conference Series, Vol. 256, No. 1, 2010, Paper 012026.

doi: $\underline{10.1088 / 1742-6596 / 256 / 1 / 012026}$ 
[61] McLean, D., "Wingtip Devices: What They Do and How They Do It," Proceedings of the Boeing Performance and Flight Operations Engineering Conference, Seattle, WA, 2005.

[62] McLean, D., Understanding Aerodynamics: Arguing from the Real Physics, Wiley, West Sussex, U.K., 2013, p. 423.

[63] Jansen, P., Perez, R. E., and Martins, J. R. R. A., "Aerostructural Optimization of Nonplanar Lifting Surfaces," Journal of Aircraft,
Vol. 47, No. 5, 2010, pp. 1491-1503.

doi: $10.2514 / 1.44727$

[64] Liem, R. P., Kenway, G. K., and Martins, J. R. R. A., "MultiPoint, Multi-Mission, High-Fidelity Aerostructural Optimization of a Long-Range Aircraft Configuration," AIAA Paper 2012-5706, Sept. 2012. 